\title{
ORTHOGONALITY AND DISJOINTNESS PRESERVING LINEAR MAPS BETWEEN FOURIER AND FOURIER-STIELTJES ALGEBRAS OF LOCALLY COMPACT GROUPS
}

\author{
ANTHONY TO-MING LAU AND NGAI-CHING WONG
}

\begin{abstract}
This paper is devoted to the study of orthogonality and disjointness preserving linear maps between Fourier and Fourier-Stieltjes algebras of locally compact groups. We show that a linear bijection $\Psi: A\left(G_{1}\right) \rightarrow A\left(G_{2}\right)$ (resp. $\left.\Psi: B\left(G_{1}\right) \rightarrow B\left(G_{2}\right)\right)$ between two Fourier algebras (resp. Fourier-Stieltjes algebras) of locally compact groups will induce a topological group isomorphism between $G_{1}$ and $G_{2}$, provided that $\Psi$ preserves both disjointness and some kind of orthogonality. This improves earlier results of J. J. Font and M. S. Monfared, where amenability of the groups or continuity of the linear maps are assumed. We also study the structure of bounded and unbounded disjointness preserving linear functionals of Fourier algebras. In the development, general results about disjointness and orthogonality preserving linear maps between $\mathrm{C}^{*}$-algebras, $\mathrm{W}^{*}$-algebras and their preduals are obtained.
\end{abstract}

\section{INTRODUCTION}

For a locally compact Hausdorff spaces $X$, let $C_{0}(X)$ denote the space of continuous complex-valued functions on $X$ vanishing at infinity. It is well known that if $X$ and $Y$ are locally compact Hausdorff spaces, then every lattice isomorphism $\Psi$ from $C_{0}(X)$ onto $C_{0}(Y)$ gives rise to a homeomorphism $\sigma$ from $Y$ onto $X$. Indeed, $\Psi(f)=\lambda \cdot f \circ \sigma$ for all $f$ in $C_{0}(X)$, where the weight function $\lambda$ is continuous and everywhere positive on $Y$ (see, e.g., $[34,1]$ ). This is also valid for the space $L^{1}(X, \mu)$ of integrable functions on a measure space $(X, \mu)$, with $\sigma$-set isomorphisms between the measure $\sigma$-algebras ([35, Proof of Theorem 3.1]; see also [40] and [49, §15]).

Date: to appear in J. Funct. Anal. (2013), http://dx.doi.org/10.1016/j.jfa.2013.04.010.

2000 Mathematics Subject Classification. 43A30, 22D25, 46J10, 46H40, 46L05.

Key words and phrases. Orthogonality preservers, disjointness preservers, locally compact groups, Fourier algebras, Fourier-Stieltjes algebras, preduals of $\mathrm{W}^{*}$-algebras.

This work is jointly supported by Canadian NSERC grant MS100, and Taiwan NSC grant 992115-M-110-007-MY3. 
A linear map between vector lattices is a lattice homomorphism if and only if it is positive and disjointness preserving (see, e.g., [3]). We are interested to see if a weaker condition on $\Psi$ ensures the equivalence of $X$ and $Y$. In $[1,5,31,21,32,25]$, it is shown that a disjointness preserving linear bijection $\Psi$ between continuous function spaces is automatically continuous and carries a similar form $\Psi(f)=\lambda \cdot f \circ \sigma$ as in the case of lattice isomorphisms.

The same is true for the group algebras of locally compact groups (see, e.g., $[22,23,20,45])$. Let $G$ be a locally compact group. Let $A(G)$ be the Fourier algebra of $G$. Then $A(G)$ is the unique predual of the group von Neumann algebra $\operatorname{VN}(G)$ of $G$. In particular, $A(G)$ carries two natural ordering. One of them is the pointwise ordering inherited from the space of bounded continuous complex-valued functions $C_{b}(G)$ and the other is the positive definite ordering induced as in the predual of a von Neumann algebra. Font [19, 20] shows that two locally compact amenable groups $G_{1}$ and $G_{2}$ are homeomorphic if there is a disjointness preserving linear bijection $\Psi$ between the Fourier algebras $A\left(G_{1}\right)$ and $A\left(G_{2}\right)$. Font's result is proved by Monfared [45], where the amenability condition is replaced with the assumption of boundedness on the linear map. However, neither the induced weight function $\lambda$ nor the homeomorphism $\sigma$ respects the group structure. In [55], Walter shows that if $\Psi: A\left(G_{1}\right) \rightarrow A\left(G_{2}\right)$ is an isometric algebra isomorphism then $G_{1}$ and $G_{2}$ are topologically and algebraically isomorphic. Using this, Arendt and De Cannière obtain the same conclusion if $\Psi$ preserves both pointwise order and positive definite order $[6,7]$. It is then natural to see what happens if $\Psi$ preserves disjointness in 'two senses': the one with pointwise ordering and the one using positive linear functionals arising from the fact that $A\left(G_{1}\right), A\left(G_{2}\right)$ are preduals of von Neumann algebras. Similarly, we also ask the same question for the Fourier-Stieltjes algebras $B\left(G_{1}\right), B\left(G_{2}\right)$.

Let $M, N$ be von Neumann algebras (or $\mathrm{W}^{*}$-algebras), and let $M_{*}, N_{*}$ denote their unique predual spaces, respectively. Following Araki [4] and Bunce and Wright [11], a linear map $\Psi: M_{*} \rightarrow N_{*}$ is said to be orthogonal decomposition preserving, or an orthogonal decomposable homomorphism if $\Psi$ preserves orthogonal decomposition of every self-adjoint normal linear functional. In other words, $\Psi(\varphi)=\Psi\left(\varphi_{+}\right)-\Psi\left(\varphi_{-}\right)$ 
is the (unique) orthogonal decomposition of $\Psi(\varphi)$ (into a difference of two disjoint positive linear functionals) whenever $\varphi=\varphi_{+}-\varphi_{-}$is the (unique) orthogonal decomposition of a self-adjoint linear functional $\varphi$ in $M_{*}$. Plainly, $\Psi$ is an orthogonal decomposable homomorphism if and only if $\Psi$ is positive, i.e., sending positive normal linear functionals to positive normal linear functionals, and orthogonality preserving on positive elements, i.e., sending orthogonal positive normal linear functionals to orthogonal normal linear functionals. In particular, $\Psi$ is automatically continuous. It is shown in [11] that $\Psi: M_{*} \rightarrow N_{*}$ is a bijective orthogonal decomposable homomorphism if and only if its dual map $\Psi^{*}: N \rightarrow M$ can be written as $\Psi^{*}=z \pi$ for a positive central invertible element $z$ in $M$ and a Jordan $*$-isomorphism $\pi$ from $N$ onto $M$.

In order to show that the disjointness and the orthogonality structures are sufficient to determine the topological group structures in the case of Fourier algebras, we need to extend both the results of Bunce and Wright [11] and Font [20] in this paper. In Section 2, we show that if a bounded bijective linear map between preduals of two $\mathrm{W}^{*}$-algebras is left or right biorthogonality preserving, then it gives rise to an algebra $*$-homomorphism. In Section 3 , we drop both amenability and boundedness assumptions and show that a disjointness preserving linear bijection between any two Fourier algebras of two locally compact groups is automatically bounded and carries a weighted composition operator form.

In Section 4, we shall present the proof of the main result in this paper:

Main Theorem. Let $G_{1}$ and $G_{2}$ be locally compact groups. Let $A\left(G_{1}\right), B\left(G_{1}\right)$ and $A\left(G_{2}\right), B\left(G_{2}\right)$ be the associated Fourier and Fourier-Stieltjes algebras of $G_{1}$ and $G_{2}$, respectively. Then, $G_{1}$ and $G_{2}$ are isomorphic as topological groups if and only if there is a bijective linear map $\Psi: A\left(G_{1}\right) \rightarrow A\left(G_{2}\right)$ or $\Psi: B\left(G_{1}\right) \rightarrow B\left(G_{2}\right)$, preserving disjointness and satisfying any one of the following conditions.

(1) $\Psi$ is orthogonal decomposition preserving.

(2) $\Psi$ is left biorthogonality preserving.

(3) $\Psi$ is right biorthogonality preserving. 
(4) $\Psi$ is left-to-right biorthogonality preserving.

(5) $\Psi$ is right-to-left biorthogonality preserving.

The proof of the Main Theorem will be based on some preliminary results in Sections 2 and 3, which have independent interests. A key step to obtain the Main Theorem is to characterize the structure of (bounded and unbounded) disjointness preserving linear functional of a Fourier algebra $A(G)$. This is done by extending the study of such functionals of an abelian $\mathrm{C}^{*}$-algebra $C_{0}(X)$ developed in [10] in Section 3. In Section 5, we explore further into the general theory of unbounded disjointness preserving linear functionals of Fourier algebras. In Section 6, applications of our technique developed in Sections 3 and 5 are demonstrated in the study of extremely left amenable semitopological semigroups.

All topologies considered in this paper are Hausdorff.

\section{Orthogonality PRESERVing Bounded Linear MAPs BetWeEn}

$\mathrm{C}^{*}$-ALGEBRAS, AND PREDUALS OF $\mathrm{W}^{*}$-ALGEBRAS

Linear orthogonality preservers between operator algebras have been well studied in past two decades. They are basically an algebra or Jordan algebra homomorphism or *-homomorphism followed by multiplications (see, e.g., [56, 51, 15, 57, 58, 12, 13, 14]). Motivated by the beautiful work of Bunce and Wright [11], we will develop in this section a theory of bounded orthogonality linear preservers between $\mathrm{C}^{*}$-algebras, and preduals of $\mathrm{W}^{*}$-algebras. In [11], orthogonality preserving bounded linear maps are always assumed to be positive. We shall relax this condition and work with $\mathrm{C}^{*}$ algebras here.

Two elements $a, b$ in a $C^{*}$-algebra are said to be orthogonal if $a^{*} b=a b^{*}=0$; in other words, $a$ and $b$ have orthogonal left and right supports. A linear map $\Phi: A \rightarrow B$ between $C^{*}$-algebras is said to be orthogonality preserving on positive elements if $\Phi(a)$ and $\Phi(b)$ are orthogonal (but not necessarily be positive) whenever $a$ and $b$ are positive and orthogonal. A linear map $\pi: A \rightarrow B$ is a Jordan homomorphism (resp. Jordan *-homomorphism) if it preserves Jordan products $\pi(a b+b a)=J(a) J(b)+J(b) J(a)$, 
or equivalently, $\pi\left(a^{2}\right)=\pi(a)^{2}$ (resp. and involutions $\left.J\left(a^{*}\right)=J(a)^{*}\right), \forall a, b \in A$. A Jordan homomorphism $\pi$ is orthogonality preserving on positive elements (see, e.g., [58, Lemma 2.1]). As shown in [33, 52], if $a b=b a$ in $A$ then $\pi(a) \pi(b)=\pi(b) \pi(a)$. In particular, $\pi$ sends central elements in $A$ to central elements in $\pi(A)$. Assume that $\pi$ is a Jordan $*$-homomorphism. Then, $\pi(A)$ is a $\mathrm{C}^{*}$-subalgebra of $B$. Moreover, $\pi\left(a b^{*} a\right)=\pi(a) \pi(b)^{*} \pi(a)$ for all $a, b$ in $A$. Consequently, $\pi$ sends partial isometries to partial isometries. When $A, B$ are $\mathrm{W}^{*}$-algebras and $\pi$ is weak* continuous, $\pi(A)$ is a $\mathrm{W}^{*}$-subalgebra of $B$.

Two normal positive linear functionals $\varphi$ and $\tau$ in $M_{*}$ are said to be orthogonal if they have orthogonal support projections $s(\varphi)$ and $s(\tau)$, or equivalently, $\|\varphi \pm \tau\|=$ $\|\varphi\|+\|\tau\|$. In general, we say two normal linear functionals $\varphi$ and $\tau$ in $M_{*}$ are left (resp. right) orthogonal if they have orthogonal left (resp. right) support projections $s_{l}(\varphi)=s(|\varphi|)$ and $s_{l}(\tau)=s(|\tau|)\left(\right.$ resp. $s_{r}(\varphi)=s\left(\left|\varphi^{*}\right|\right)$ and $\left.s_{r}(\tau)=s\left(\left|\tau^{*}\right|\right)\right)$. Here, $\varphi^{*}(x)=\overline{\varphi\left(x^{*}\right)}$ (complex conjugate) and $|\varphi|$ is the absolute value of $\varphi$. Note that $s_{l}(\varphi)$ and $\left.s_{r}(\varphi)\right)$ are the smallest projections in $M$ such that

$$
\varphi(x)=\varphi\left(s_{l}(\varphi) x\right)=\varphi\left(x s_{r}(\varphi)\right), \quad \forall x \in M .
$$

Bunce and Wright show:

Proposition 2.1 ([11, Corollary 2.9]). Let $M$ and $N$ be $W^{*}$-algebras, and let $\Psi$ : $M_{*} \rightarrow N_{*}$ be a bounded bijective linear map. Then the following are equivalent.

(1) $\Psi: M_{*} \rightarrow N_{*}$ is orthogonal decomposition preserving, i.e., $\Psi$ is positive and orthogonality preserving on positive elements of $M_{*}$.

(2) $\Psi^{*}: N \rightarrow M$ is orthogonal decomposition preserving, i.e., $\Psi^{*}$ is positive and orthogonality preserving on positive elements of $N$.

(3) $\Psi^{*}=z \pi$, where $z=\Psi^{*}(1)$ is a positive invertible central element in $M$ and $\pi: N \rightarrow M$ is a surjective weak ${ }^{*}$ continuous Jordan $*$-isomorphism.

Recall that the multiplier algebra $M(B)$ of a $\mathrm{C}^{*}$-algebra $B$ can be identified with the $\mathrm{C}^{*}$-subalgebra of $B^{* *}$ as

$$
M(B)=\left\{x \in B^{* *}: x B+B x \subset B\right\} .
$$


Write $Z(B)$ and $Z(M(B))$ for the centers of $B$ and $M(B)$, respectively. Let $\Phi: A \rightarrow$ $B$ be a bounded linear map between $\mathrm{C}^{*}$-algebra. For simplicity of notations, we write again $\Phi$ for the bidual map $\Phi^{* *}: A^{* *} \rightarrow B^{* *}$, which is also weak ${ }^{*}$-weak* continuous.

The following is a $\mathrm{C}^{*}$-algebra version of $[11$, Proposition 1.1] that we shall need. However, rather than assuming $\Phi$ to be positive, we require that $\Phi$ has a dense range. Meanwhile, we also extend the results in [57], where $\Phi(1)\left(=\Phi^{* *}(1)\right)$ is assumed to be a partial isometry and $\Phi$ is assumed to preserve all rather than just positive orthogonal pairs.

Theorem 2.2. Let $\Phi: A \rightarrow B$ be a bounded linear map between two $C^{*}$-algebras $A$ and $B$ with dense range. Then $\Phi$ is orthogonality preserving on positive elements if and only if

$$
\Phi=\Phi(1) \pi
$$

where $\Phi(1) \in M(B)$ with $\Phi(1)^{*} \Phi(1)=\Phi(1) \Phi(1)^{*} \in Z(M(B))$ and $\pi: A \rightarrow M(B)$ is a Jordan *-homomorphism, such that $\Phi(1) \pi(A) \subseteq B$.

Moreover, $\Phi(1)$ is invertible if and only if $\Phi$ is surjective. In this case, $\pi(A)=B$.

Proof. The sufficiency is trivial, and we will show the necessity below.

Let $a \in A^{+}$with spectrum $X \subseteq[0,\|a\|]$. Identify the abelian $\mathrm{C}^{*}$-subalgebra of $A^{* *}$ generated by 1 and $a$ with $C(X)$. Let $0=\alpha_{0}<\alpha_{1}<\cdots<\alpha_{n-1}<\alpha_{n}=\|a\|+1$, and $X=\bigcup_{k} X_{k}$ be the partition of $X$ with $X_{k}=X \cap\left[\alpha_{k-1}, \alpha_{k}\right)$. In particular, $1=\sum_{k} 1_{X_{k}}$, where $1_{X_{k}}$ is the characteristic function of $X_{k}$ for $k=1, \ldots, n$. Note that some $X_{k}$ might be empty, and thus $1_{X_{k}}=0$ in these cases. Indeed, $1_{X_{k}}$ is the atomic part of the spectral projection $E_{a}\left(X_{k}\right)$ of $a$ for the subset $X_{k}$ of its spectrum. Below, we shall identify $f(t)=t$ with $a$ and $1_{X_{k}}$ with $E_{a}\left(X_{k}\right)$ as (atomic parts of universally measurable) elements of $A^{* *}$. Note that $\Phi$ sends universally measurable elements to universally measurable elements, and universally measurable elements of $A^{* *}$, including spectral projections of elements of $A$, are determined by their atomic parts (see, e.g., [47, Section 4.3]). 
For $1<j<k$, we can find two sequences of continuous non-negative functions $\left\{f_{n}\right\}_{n}$ and $\left\{g_{n}\right\}_{n}$ from $C(X) \cap A$ such that $f_{m} g_{m+p}=0$ for all $m, p=1,2, \ldots$, $f_{m} \rightarrow 1_{X_{j}}$, and $g_{m} \rightarrow 1_{X_{k}}$ pointwisely on $X$, and hence in the weak* topology of $A^{* *}$. By the weak* continuity of $\Phi\left(=\Phi^{* *}\right)$, we see that

$$
\Phi\left(1_{X_{k}}\right) \Phi\left(f_{m}\right)^{*}=\lim _{p \rightarrow \infty} \Phi\left(g_{m+p}\right) \Phi\left(f_{m}\right)^{*}=0, \quad \forall m=1,2, \ldots
$$

Thus

$$
\Phi\left(1_{X_{k}}\right) \Phi\left(1_{X_{j}}\right)^{*}=\lim _{m \rightarrow \infty} \Phi\left(1_{X_{k}}\right) \Phi\left(f_{m}\right)^{*}=0, \quad \forall 1<j<k .
$$

Similarly, we have

$$
\Phi\left(1_{X_{k}}\right)^{*} \Phi\left(1_{X_{j}}\right)=0, \quad \forall 1<j<k .
$$

When $j=1$, set $X_{1 m}=X_{1} \cap\left[0, \frac{m \alpha_{1}}{m+1}\right.$ ). As the (atomic part of the) spectral projection $E_{a}\left(X_{1 m}\right)$ of $a$ for the open subset $X_{1 m}$ of its spectrum, $1_{X_{1 m}}$ is (the atomic part of) an open projection in $A^{* *}$ for $m=1,2, \ldots$ Hence, there is an increasing net $\left\{a_{\lambda}\right\}_{\lambda}$ in $A^{+}$converging to $1_{X_{1 m}}$ in the weak* topology of $A^{* *}$ (see, e.g., [47, Proposition 3.11.9] or [9]). Using an argument similar to above, we get

$$
\Phi\left(1_{X_{k}}\right) \Phi\left(1_{X_{1 m}}\right)^{*}=\Phi\left(1_{X_{k}}\right)^{*} \Phi\left(1_{X_{1 m}}\right)=0, \quad \forall k=2, \ldots, n, m=1,2, \ldots
$$

Letting $m \rightarrow \infty$, we have

$$
\Phi\left(1_{X_{k}}\right) \Phi\left(1_{X_{1}}\right)^{*}=\Phi\left(1_{X_{k}}\right)^{*} \Phi\left(1_{X_{1}}\right)=0 .
$$

Consequently, for each $j=1,2, \ldots, n$, we have

$$
\begin{aligned}
& \Phi(1) \Phi\left(1_{X_{j}}\right)^{*}=\Phi\left(1_{X_{j}}\right) \Phi\left(1_{X_{j}}\right)^{*}, \\
& \Phi(1)^{*} \Phi\left(1_{X_{j}}\right)=\Phi\left(1_{X_{j}}\right)^{*} \Phi\left(1_{X_{j}}\right),
\end{aligned}
$$

for all $j=1, \ldots, n$. Note that $a$ can be approximated in norm by step functions in the form of $\sum_{j} \lambda_{j} 1_{X_{j}}$. Thus, $\Phi(a)$ can be approximated in norm by $\sum_{j} \lambda_{j} \Phi\left(1_{X_{j}}\right)$. It follows from (1) and (2) that

$$
\begin{aligned}
& \Phi(1) \Phi\left(a^{2}\right)^{*}=\Phi(a) \Phi(a)^{*}=\Phi\left(a^{2}\right) \Phi(1)^{*}, \\
& \Phi(1)^{*} \Phi\left(a^{2}\right)=\Phi(a)^{*} \Phi(a)=\Phi\left(a^{2}\right)^{*} \Phi(1),
\end{aligned}
$$

for all positive $a$ in $A$. Since $\Phi$ has a dense range, we see that $\Phi(1) \in M(B)$. 
Let $p$ be a projection in $A^{* *}$. Let $\left\{a_{\lambda}\right\}_{\lambda}$ be a net from $A^{+}$such that $a_{\lambda} \rightarrow p$ in the weak* topology. Then (3) and (4) imply

$$
\begin{aligned}
& \Phi(1) \Phi(p)^{*}=\Phi(p) \Phi(p)^{*}=\Phi(p) \Phi(1)^{*}, \\
& \Phi(1)^{*} \Phi(p)=\Phi(p)^{*} \Phi(p)=\Phi(p)^{*} \Phi(1) .
\end{aligned}
$$

Consequently,

$$
\Phi(1) \Phi(1)^{*} \Phi(p)=\Phi(1) \Phi(p)^{*} \Phi(1)=\Phi(p) \Phi(1)^{*} \Phi(1)
$$

This implies in turn that

$$
\Phi(1) \Phi(1)^{*} \Phi(a)=\Phi(a) \Phi(1)^{*} \Phi(1), \quad \forall a \in A
$$

by the weak* continuity of $\Phi\left(=\Phi^{* *}\right)$. Therefore, the positive element

$$
\left(\Phi(1)^{*} \Phi(a)\right)^{*}\left(\Phi(1)^{*} \Phi(a)\right)=\Phi(a)^{*} \Phi(1) \Phi(1)^{*} \Phi(a)=\Phi(a)^{*} \Phi(a) \Phi(1)^{*} \Phi(1) .
$$

Hence,

$$
\Phi(a)^{*} \Phi(a) \Phi(1)^{*} \Phi(1)=\Phi(1)^{*} \Phi(1) \Phi(a)^{*} \Phi(a), \quad \forall a \in A
$$

Since $\Phi$ has a dense range, $\Phi(1)^{*} \Phi(1)$ is a central element. By (7), we see that $\Phi(1)^{*} \Phi(1)=\Phi(1) \Phi(1)^{*}$. By (5) and (6), the support projection of $\Phi(1)$ is $e$, the identity element of $B^{* *}$.

Suppose that $\Phi(1)$ is invertible in $M(B)$ and $b \Phi(1)=e$ for some element $b$ in $M(B)$. Inherited from $\Phi(1)$, we have $b^{*} b=b b^{*} \in Z(M(B))$. The bounded linear map $\pi: A \rightarrow B$ defined by $\pi(a)=b \Phi(a)$ preserves again orthogonality of selfadjoint elements. Moreover, $\pi(1)=e$. Here, again we write $\pi$ for the bidual map $\pi^{* *}: A^{* *} \rightarrow B^{* *}$. By (5) and (6), we have

$$
\pi(p)=\pi(p)^{*} \pi(p) \text { and } \pi(p)^{*}=\pi(p) \pi(p)^{*},
$$

and hence

$$
\pi(p)=\pi(p)^{*}=\pi(p)^{2}
$$

for all projections $p$ in $A^{* *}$. This ensures that $\pi$ is a Jordan $*$-homomorphism from $A$ into $B$. Note that $\Phi=\Phi(1) \pi$. Since $\Phi$ has a dense range and $\pi(A)$ is a $\mathrm{C}^{*}$-algebra, $\pi$ is surjective. Furthermore, for any $y$ in $B$, we have $\Phi(1)^{-1} y \in B$ and thus there 
is an $a$ in $A$ with $\pi(a)=\Phi(1)^{-1} y$. This gives $\Phi(a)=\Phi(1) \pi(a)=y$, and hence $\Phi$ is surjective.

In the case that $\Phi(1)$, or equivalently the central multiplier $|\Phi(1)|=\sqrt{\Phi(1)^{*} \Phi(1)}$, is not invertible, we identify the abelian $\mathrm{W}^{*}$-subalgebra $Z\left(B^{* *}\right)$ with $C(Y)$ and $|\Phi(1)|$ with an $f \geq 0$ in $C(Y)$, where $Y$ is a compact hyperstonean space. Since the support projection of $|\Phi(1)|$ is $e$, we see that $\{y \in Y: f(y)>0\}$ is dense in $Y$. For each $n=1,2, \ldots$, let $K_{n}$ be the closure in $Y$ of the open set $\{y \in Y: f(y)>1 / n\}$. Then each $K_{n}$ is a clopen subset of $Y$. Let $e_{n}$ be the central projection in $B^{* *}$ arising from the characteristic function of $K_{n}$. Clearly, $\left\{e_{n}\right\}$ is increasing with $e_{n} \uparrow e$, and $e_{n} \Phi(1)$ is invertible in $e_{n} B^{* *}$. As discussed above, for the linear orthogonality preserving map $e_{n} \Phi$ from $A$ into the $\mathrm{C}^{*}$-subalgebra $\overline{e_{n} B}$ of $e_{n} B^{* *}$ there is a Jordan *-homomorphism $\pi_{n}: A \rightarrow e_{n} B^{* *}$ such that $e_{n} \Phi(a)=e_{n} \Phi(1) \pi_{n}(a), \forall a \in A$. As $e=e_{1}+\sum_{n \geq 1}\left(e_{n+1}-e_{n}\right)$, we have $\Phi=\Phi(1) \pi$ where $\pi: A \rightarrow B^{* *}$ is a Jordan $*$-homomorphism defined by $\pi(a)=e_{1} \pi_{1}(a)+\sum_{n \geq 1}\left(e_{n+1}-e_{n}\right) \pi_{n}(a), \forall a \in A$. Since $\pi(A)$ is a $C^{*}$-algebra and $\Phi(A)$ is dense in $B$, for any $a$ in $A$ it follows from

$$
\Phi(A) \pi(a)=\Phi(1) \pi(A) \pi(a) \subseteq \Phi(1) \pi(A)=\Phi(A) \subseteq B
$$

that $\pi(a)$ is a right multiplier of $B$. Because $\pi(a)^{*}=\pi\left(a^{*}\right)$ is also a right multiplier, $\pi(a)$ is a multiplier of $B$. That is, $\pi$ sends $A$ into $M(B)$.

Finally, assume that $\Phi(A)=B$. Let $\left\{e_{\lambda}\right\}$ be an increasing positive approximate identity in $B$. It follows from the open mapping theorem that there is a uniformly bounded net $\left\{a_{\lambda}\right\}$ in $A$ such that $e_{\lambda}=\Phi\left(a_{\lambda}\right)=\Phi(1) \pi\left(a_{\lambda}\right)$. Let $b$ be a weak* cluster point of the bounded net $\left\{\pi\left(a_{\lambda}\right)\right\}$ in $B^{* *}$. We have $e=\Phi(1) b$, and thus $\Phi(1)$ is invertible. As already discussed above, $\pi$ maps $A$ onto $B$ in this case.

Example 2.3. (a) Let $\Phi: C[0,1] \rightarrow C_{0}(0,1]$ be the orthogonality preserving bounded linear map defined by $\Phi(f)(t)=t \exp (i / t) f(t)$. Then $\Phi$ is not positive and the range of $\Phi$ is not self-adjoint. Anyway, $\Phi$ has a dense range, and the conclusion of Theorem 4.1 holds. Note that the associative Jordan $*$-homomorphism $\pi(f)=f$ maps $C[0,1]$ outside $C_{0}(0,1]$. 
(b) Consider the bijective linear map $\Phi: M_{2} \rightarrow M_{2}$ between $2 \times 2$ complex matrices defined by

$$
\Phi\left(\begin{array}{ll}
a & b \\
c & d
\end{array}\right)=\left(\begin{array}{ll}
0 & 1 \\
1 & 0
\end{array}\right)\left(\begin{array}{ll}
a & b \\
c & d
\end{array}\right)=\left(\begin{array}{ll}
c & d \\
a & b
\end{array}\right) .
$$

Then $\Phi$ is orthogonality preserving with $\Phi(1)^{*} \Phi(1)=\Phi(1) \Phi(1)^{*}=1$. But $\Phi(1)$ is not central.

In [11], $\Phi: M \rightarrow N$ is assumed to be a weak* continuous orthogonality preserving map between two $\mathrm{W}^{*}$-algebras sending positive elements to positive elements. The following two results extend [11, Theorem 2.1].

Corollary 2.4. Let $\Phi: M \rightarrow N$ be a weak* continuous linear map between two $W^{*}$-algebras $M$ and $N$ with dense range. Then $\Phi$ is orthogonality preserving on positive elements if and only if $\Phi(1)^{*} \Phi(1)=\Phi(1) \Phi(1)^{*}$ is central and there is a weak ${ }^{*}$ continuous surjective Jordan $*$-homomorphism $\pi: M \rightarrow N$ such that

$$
\Phi=\Phi(1) \pi
$$

Proof. Arguing as in the proof of Theorem 2.2, we can work within $M, N$ without referring to $M^{* *}, N^{* *}$ or the bidual map $\Phi^{* *}$. Moreover, $\pi(M)=N$ as $\Phi$ has a dense range.

Corollary 2.5. Let $\Phi: M \rightarrow N$ be a weak ${ }^{*}$ continuous linear map between $W^{*}$ algebras with $\Phi(1) \geq 0$. Then $\Phi$ is orthogonality preserving on positive elements if and only if there is a weak ${ }^{*}$ continuous Jordan $*$-homomorphism $\pi: M \rightarrow N$ and a central element $z$ in $M$ such that

$$
\Phi=\pi(z \cdot)
$$

Proof. We verify the sufficiency only. Let $N_{\Phi}$ be the $\mathrm{W}^{*}$-subalgebra of $N$ generated by the range of $\Phi$. We argue as in the proof of Theorem 2.2 by considering $\Phi$ as a map from $M$ into $N_{\Phi}$. Since $\Phi(1) \geq 0$, it follows from (7) that $\Phi(1)=|\Phi(1)|$ is a central element of $N_{\Phi}$. We can then obtain a surjective Jordan homomorphism $\pi: M \rightarrow N_{\Phi}$ such that $\Phi=\theta(1) \pi$. Thus there is a central element $z$ in $M$ such that $\pi(z)=\Phi(1)$ ([54]). Consequently, $\Phi(a)=\theta(1) \pi(a)=\pi(z) \pi(a)=\pi(z a), \forall a \in A$. 
We can now sharpen Proposition 2.1 by reducing the positivity assumption on $\Psi^{*}(x) \geq 0$ for all $x \geq 0$ to a single condition $\Psi^{*}(1) \geq 0$.

Theorem 2.6. Let $M, N$ be $W^{*}$-algebras with preduals $M_{*}, N_{*}$, respectively. Let $\Psi: M_{*} \rightarrow N_{*}$ be a bounded bijective linear map. Then the following are equivalent.

(1) $\Psi: M_{*} \rightarrow N_{*}$ is orthogonal decomposition preserving, i.e., $\Psi$ is positive and orthogonality preserving on positive elements of $M_{*}$.

(2) $\Psi^{*}: N \rightarrow M$ is orthogonality preserving on positive elements, and $\Psi^{*}(1) \geq 0$.

(3) $\Psi^{*}=z \pi$, where $z=\Psi^{*}(1)$ is a positive invertible central element in $M$ and $\pi: N \rightarrow M$ is a surjective weak* continuous Jordan $*$-isomorphism.

In the case $\Psi=\Phi^{*}$ is the dual map of a bounded bijective linear map $\Phi: A \rightarrow B$ between two $C^{*}$-algebras, the above statements are also equivalent to

(4) $\Phi$ is orthogonality preserving on positive elements, and $\Phi^{* *}(1) \geq 0$.

(5) $\Phi=z \pi$, where $z=\Phi^{* *}(1)$ is a positive invertible central multiplier of $B$ and $\pi: A \rightarrow B$ is a Jordan *-isomorphism.

Proof. The equivalence between (1) and (3) follows from Proposition 2.1, while that between (2) and (3) follows from Theorem 2.2 and its corollaries. If $A, B$ are $\mathrm{C}^{*}$ algebras, then $A^{*}, B^{*}$ are preduals of the $\mathrm{W}^{*}$-algebras $N=A^{* *}, M=B^{* *}$, respectively. It is plain that (2) implies (4), and (5) implies (3). Finally, it follows from Theorem 2.2 that (4) implies (5).

Next, we will develop a corresponding part of Theorem 2.6 for non-positive one-side linear orthogonality preservers of normal linear functionals. Let $M$ be a $\mathrm{W}^{*}$-algebra with predual $M_{*}$. Write $s(\varphi)$ for the support projection of a normal positive linear functional $\varphi$ in $M_{*}$. It is known that a projection $p$ in $M$ is $\sigma$-finite if and only if it is of the form $s(\varphi)$ for some positive normal linear functional $\varphi$ in $M_{*}$ (see, e.g., [42, Proposition 1.14.7]). In general, every projection $p$ in $M$ is an orthogonal sum of $\sigma$-finite projections (see, e.g., [42, Proposition 1.14.10]). 
Lemma 2.7. Let $M$ and $N$ be $W^{*}$-algebras with preduals $M_{*}, N_{*}$, respectively. Let $\Psi: M_{*} \rightarrow N_{*}$ be a bounded bijective linear map. Then the following conditions are equivalent.

(1) $\Psi$ is right biorthogonality preserving, i.e.,

$$
s_{r}(\varphi) s_{r}(\tau)=0 \quad \Longleftrightarrow \quad s_{r}(\Psi(\varphi)) s_{r}(\Psi(\tau))=0, \quad \forall \varphi, \tau \in M_{*} .
$$

(2) $\Psi^{*}(1)$ is an invertible element and there is a weak* continuous algebra $*$-isomorphism $\pi: N \rightarrow M$ such that

$$
\Psi^{*}=\Psi^{*}(1) \pi
$$

In this case, if we write $\pi_{\Psi}: N \rightarrow M$ for the inverse of $\pi$ and write $z=\Psi^{*}(1)$ then

$$
\Psi^{*}\left(\pi_{\Psi}(x)\right)=z x, \quad \forall x \in M
$$

Proof. Let $z=\Psi^{*}(1)$ and $\varphi^{\prime}=\Phi(\varphi)$ for each $\varphi$ in $M_{*}$.

(2) $\Longrightarrow(1)$. Observe that for any $\varphi$ in $M_{*}$ we have

$$
\begin{aligned}
\varphi^{\prime}\left(x \pi_{\Psi}\left(s_{r}(\varphi)\right)\right) & =\varphi\left(\Psi^{*}\left(x \pi_{\Psi}\left(s_{r}(\varphi)\right)\right)\right)=\varphi\left(z \pi\left(x \pi_{\Psi}\left(s_{r}(\varphi)\right)\right)\right) \\
& =\varphi\left(z \pi(x) s_{r}(\varphi)\right)=\varphi(z \pi(x)) \\
& =\varphi^{\prime}(x), \quad \forall x \in M .
\end{aligned}
$$

Hence, $s_{r}\left(\varphi^{\prime}\right) \leq \pi_{\Psi}\left(s_{r}(\varphi)\right)$. Conversely,

$$
\begin{aligned}
\varphi\left(x \pi\left(s_{r}\left(\varphi^{\prime}\right)\right)\right) & =\varphi\left(z\left(z^{-1} x \pi\left(s_{r}\left(\varphi^{\prime}\right)\right)\right)\right)=\varphi\left(\Psi^{*}\left(\pi_{\Psi}\left(z^{-1} x \pi\left(s_{r}\left(\varphi^{\prime}\right)\right)\right)\right)\right) \\
& =\varphi^{\prime}\left(\pi_{\Psi}\left(z^{-1} x\right) s_{r}\left(\varphi^{\prime}\right)\right)=\varphi^{\prime}\left(\pi_{\Psi}\left(z^{-1} x\right)\right) \\
& =\varphi\left(\Psi^{*}\left(\pi_{\Psi}\left(z^{-1} x\right)\right)\right)=\varphi\left(z\left(z^{-1} x\right)\right) \\
& =\varphi(x), \quad \forall x \in M .
\end{aligned}
$$

Hence, $s_{r}(\varphi) \leq \pi\left(s_{r}\left(\varphi^{\prime}\right)\right)$, and thus $s_{r}\left(\varphi^{\prime}\right)=\pi_{\Psi}\left(s_{r}(\varphi)\right)$. Consequently, $\Psi$ is right biorthogonality preserving.

(1) $\Longrightarrow$ (2). For any $\tau$ in $M_{*}$, let $\tau_{1}=\tau\left(\cdot\left(1-s_{r}(\varphi)\right)\right)$. Then $s_{r}\left(\tau_{1}\right) s_{r}(\varphi)=0$. Hence $s_{r}\left(\tau_{1}^{\prime}\right) s_{r}\left(\varphi^{\prime}\right)=0$ as well. This gives $0=\tau_{1}^{\prime}\left(x s_{r}\left(\varphi^{\prime}\right)\right)=\tau_{1}\left(\Psi^{*}\left(x s_{r}\left(\varphi^{\prime}\right)\right)=\tau\left(\Psi^{*}\left(x s_{r}\left(\varphi^{\prime}\right)\right)\left(1-s_{r}(\varphi)\right)\right), \quad \forall x \in N, \forall \tau \in M_{*}\right.$. 
Consequently,

$$
\Psi^{*}\left(x s_{r}\left(\varphi^{\prime}\right)\right)=\Psi^{*}\left(x s_{r}\left(\varphi^{\prime}\right)\right) s_{r}(\varphi), \quad \forall x \in N, \forall \varphi \in M_{*} .
$$

Write $1=s_{r}(\varphi)+\sum_{i} s_{r}\left(\tau_{i}\right)$ as an orthogonal sum of $\sigma$-finite projections in $M$. Assume the orthogonal sum $s_{r}\left(\varphi^{\prime}\right)+\sum_{i} s_{r}\left(\tau_{i}^{\prime}\right)=1-q$ and $q=\sum_{j} s_{r}\left(\zeta_{j}^{\prime}\right)$ for some $\zeta_{j}$ in $M_{*}$. Since $\Phi^{-1}$ also preserves right orthogonality, $s_{r}\left(\zeta_{j}\right) s_{r}(\varphi)=s_{r}\left(\zeta_{j}\right) s_{r}\left(\tau_{i}\right)=0, \forall i, j$. It follows $\zeta_{j}=0, \forall j$. In other words, $q=0$ and $1=s_{r}\left(\varphi^{\prime}\right)+\sum_{i} s_{r}\left(\tau_{i}^{\prime}\right)$ in $N$. Now, (8) provides

$$
\Psi^{*}\left(x s_{r}\left(\tau_{i}^{\prime}\right)\right) s_{r}(\varphi)=\Psi^{*}\left(x s_{r}\left(\tau_{i}^{\prime}\right)\right) s_{r}\left(\tau_{i}\right) s_{r}(\varphi)=0, \quad \forall i
$$

and thus

$$
\begin{aligned}
\Psi^{*}\left(x s_{r}\left(\varphi^{\prime}\right)\right) & =\Psi^{*}\left(x s_{r}\left(\varphi^{\prime}\right)\right) s_{r}(\varphi)+\sum_{i} \Psi^{*}\left(x s_{r}\left(\tau_{i}^{\prime}\right)\right) s_{r}(\varphi) \\
& =\Psi^{*}(x) s_{r}(\varphi), \quad \forall x \in N, \forall \varphi \in M_{*} .
\end{aligned}
$$

In particular, setting $x=1$ we have

$$
\Psi^{*}\left(s_{r}\left(\varphi^{\prime}\right)\right)=\Psi^{*}(1) s_{r}(\varphi), \quad \forall \varphi \in M_{*} .
$$

We use an argument similar to the one given in [11, Theorem 2.6] to obtain the representation $\Psi^{*}\left(\pi_{\Psi}(x)\right)=\Psi^{*}(1) x$. Let $x \in M$. We claim there is an element $x^{\prime}$ in $N$ such that $\Psi^{*}\left(x^{\prime}\right)=\Psi^{*}(1) x$. Such an $x^{\prime}$ is necessarily unique as $\Psi^{*}$ is injective. Let $p$ be any projection in $M$, and write $p=\sum s_{r}(\zeta)$ as an orthogonal sum of right support projections. Since $\Psi$ preserves right orthogonality, $p^{\prime}=\sum s_{r}\left(\zeta^{\prime}\right)$ is a projection in $N$. By the weak* continuity of $\Psi^{*}$ and (10), we have

$$
\Psi^{*}\left(p^{\prime}\right)=\sum \Psi^{*}\left(s_{r}\left(\zeta^{\prime}\right)\right)=\sum \Psi^{*}(1) s_{r}(\zeta)=\Psi^{*}(1) p .
$$

Now let $x \in M$ with $0 \leq x \leq 1$. We can write $x=\sum\left(p_{n} / 2^{n}\right)$, for certain spectral projections $p_{n}$ of $x$. Let $p_{n}^{\prime}$ be a projection in $N$ such that $\Psi^{*}\left(p_{n}^{\prime}\right)=\Psi^{*}(1) p_{n}$ for each $n$. Thus $x^{\prime}=\sum\left(p_{n}^{\prime} / 2^{n}\right) \in N$ and

$$
\Psi^{*}(1) x=\sum \Psi^{*}(1) \frac{p_{n}}{2^{n}}=\sum \frac{\Psi^{*}\left(p_{n}^{\prime}\right)}{2^{n}}=\Psi^{*}\left(x^{\prime}\right) .
$$

This shows the claim.

Define $\pi_{\Psi}: M \rightarrow N$ by $\pi_{\Psi}(x)=x^{\prime}$ as above. It is easy to see that $\pi_{\Psi}$ is a weak* continuous Jordan $*$-homomorphism satisfying that 
(a) $\Psi^{*}\left(\pi_{\Psi}(x)\right)=\Psi^{*}(1) x$, for all $x$ in $M$.

(b) $\pi_{\Psi}\left(s_{r}(\zeta)\right)=s_{r}\left(\zeta^{\prime}\right)$, for all $\zeta$ in $M_{*}$.

Since the range of $\pi_{\Psi}$ is a $\mathrm{W}^{*}$-subalgebra of $N$ containing all $s_{r}\left(\zeta^{\prime}\right)$, we have $\pi_{\Psi}(M)=$ $N$. Therefore, $\pi_{\Psi}$ maps $M$ isomorphically onto $N$ as Jordan $*$-algebras. Since $\Psi^{*}(1) M=\Psi^{*}\left(\pi_{\Psi}(M)\right)=\Psi^{*}(N)=M$, we see that $\Psi^{*}(1)$ is invertible.

Moreover, it follows from (9) and the argument deriving (a) above that for all $y$ in $N$ we have

$$
\Psi^{*}\left(\pi_{\Psi}(x) \pi_{\Psi}(y)\right)=\Psi^{*}\left(\pi_{\Psi}(x)\right) y=\Psi^{*}(1) x y=\Psi^{*}\left(\pi_{\Psi}(x y)\right), \quad \forall x \in N
$$

By the injectivity of $\Psi^{*}$ we have $\pi_{\Psi}(x) \pi_{\Psi}(y)=\pi_{\Psi}(x y)$ for all $x, y$ in $M$. So $\pi_{\Psi}$ is a weak*-continuous algebra $*$-isomorphism.

Let $\pi: N \rightarrow M$ be the inverse of $\pi_{\Psi}$. Then $\Psi^{*}=\Psi^{*}(1) \pi$ on $N$.

We can also define left orthogonality preserving property for a bijective linear map $\varphi \mapsto \varphi^{\prime}$ between preduals of $\mathrm{W}^{*}$-algebras. Even more general, we can define leftto-right and right-to-left orthogonality preservers. Namely, they are those sending normal linear functionals with disjoint left (resp. right) support projections to normal linear functionals with disjoint right (resp. left) support projections. Note that a linear map $\Psi: M_{*} \rightarrow N_{*}$ is left (resp. right, left-to-right, right-to-left) orthogonality preserving if and only if

$$
\begin{array}{rll}
s(|\varphi|) s(|\tau|)=0 & \Longrightarrow & s\left(\left|\varphi^{\prime}\right|\right) s\left(\left|\tau^{\prime}\right|\right)=0 \\
\left(\text { resp. } s\left(\left|\varphi^{*}\right|\right) s\left(\left|\tau^{*}\right|\right)=0\right. & \Longrightarrow & s\left(\left|\left(\varphi^{\prime}\right)^{*}\right|\right) s\left(\left|\left(\tau^{\prime}\right)^{*}\right|\right)=0, \\
s(|\varphi|) s(|\tau|)=0 & \Longrightarrow & s\left(\left|\left(\varphi^{\prime}\right)^{*}\right|\right) s\left(\left|\left(\tau^{\prime}\right)^{*}\right|\right)=0, \\
s\left(\left|\varphi^{*}\right|\right) s\left(\left|\tau^{*}\right|\right)=0 & \Longrightarrow & \left.s\left(\left|\left(\varphi^{\prime}\right)\right|\right) s\left(\left|\left(\tau^{\prime}\right)\right|\right)=0\right) .
\end{array}
$$

In particular, if the map is positive, i.e., $\varphi \geq 0 \Longrightarrow \varphi^{\prime} \geq 0$, then all left, right, left-to-right and right-to-left orthogonality preserving properties coincide on positive elements with the orthogonal decomposition preserving property of Bunce and Wright $[11]$. 
The following theorem supplements a result of Bunce and Wright (Proposition 2.1, and see Theorem 2.6). It provides another positive answer to a question of Araki [4]. Here we do not assume the orthogonality preserving maps are positive.

Theorem 2.8. Let $M$ and $N$ be $W^{*}$-algebras with preduals $M_{*}$ and $N_{*}$, respectively. Let $\Psi: M_{*} \rightarrow N_{*}$ be a bounded bijective linear map. The following are all equivalent.

(1) $\Psi$ is right biorthogonality preserving, i.e.,

$$
s_{r}(\varphi) s_{r}(\tau)=0 \Longleftrightarrow s_{r}(\Psi(\varphi)) s_{r}(\Psi(\tau))=0, \quad \forall \varphi, \tau \in M_{*} .
$$

(2) $\Psi^{*}$ is right orthogonality preserving, i.e.,

$$
a b^{*}=0 \Longrightarrow \Psi^{*}(a)\left(\Psi^{*}(b)\right)^{*}=0, \quad \forall a, b \in M .
$$

(3) $\Psi^{*}=z \pi$, where $z=\Psi^{*}(1)$ is an invertible element in $M$ and $\pi: N \rightarrow M$ is a weak* $^{*}$ continuous algebra $*$-isomorphism.

In the case $\Psi=\Phi^{*}$ is the dual map of a bounded bijective linear map $\Phi: A \rightarrow B$ between two $C^{*}$-algebras, the above statements are also equivalent to

(4) $\Phi$ is right orthogonality preserving.

(5) $\Phi=z \pi$, where $z=\Phi^{* *}(1)$ is an invertible multiplier of $B$ and $\pi: A \rightarrow B$ is an algebra $*$-isomorphism.

Proof. The implications (3) implying (1) and (1) implying (2) follow from Lemma 2.7, while that (2) implying (3) follows from Schweizer [51, Theorem 4.7], see also [41, Theorem 1.3(c)]. If $A, B$ are $\mathrm{C}^{*}$-algebras, then $A^{*}, B^{*}$ are preduals of the $\mathrm{W}^{*}$ algebras $N=A^{* *}, M=B^{* *}$, respectively. The implication from (2) to (4) is clear. The implication from (4) to (5) is due to Schweizer [51, Theorem 4.7] again. Finally, the implication from (5) to (3) is trivial.

Remark 2.9. Theorem 2.8 has corresponding 'left', 'left-to-right' and 'right-to-left' versions, too. But $\Psi^{*}$ is right-to-left (resp. left-to-right) orthogonality preserving whenever $\Psi$ is left-to-right (resp. right-to-left) biorthogonality preserving, and $\pi$ is an algebra $*$-anti-isomorphisms in these cases. Moreover, $\Psi^{*}(\cdot)=\pi(\cdot) z$ for left and left-to-right preservers. 
Let $G$ be a topological group, i.e., $G$ is a group with a Hausdorff topology, such that the mappings $x \mapsto x^{-1}$ from $G$ into $G$ and $(x, y) \mapsto x y$ from $G \times G$ into $G$ are continuous. Let $P(G)$ denote the collection of all continuous positive definite functions on $G$, i.e., the collection of all continuous complex valued functions $f$ on $G$ such that for any complex numbers $\lambda_{1}, \ldots, \lambda_{n}$ and any $a_{1}, \ldots, a_{n}$ in $G$, we have

$$
\sum_{i=1}^{n} \sum_{j=1}^{n} \overline{\lambda_{i}} \lambda_{j} f\left(a_{i}^{-1} a_{j}\right) \geq 0 .
$$

Let $B(G)$ denote the linear span of $P(G)$. As shown in [36], $B(G)$ can be identified with the predual of a von Neumann algebra $W^{*}(G) \subset B\left(\mathcal{H}_{\omega}\right)$, where $\omega$ is a *-homomorphism of $G$ into the group of unitary operators in $B\left(\mathcal{H}_{\omega}\right)$, the space of bounded linear operators from a Hilbert space $\mathcal{H}_{\omega}$ into $\mathcal{H}_{\omega}$. Furthermore, $B(G)$, with the predual norm of $W^{*}(G)$ is a commutative Banach algebra called the FourierStieltjes algebra of $G$. (See also [37].)

Theorem 2.8 can be applied to obtain the following characterization of bounded orthogonality preserving maps between $B(G)$ and $B(H)$ of two topological groups.

Corollary 2.10. Let $G$ and $H$ be topological groups, and $\Psi: B(G) \rightarrow B(H)$ be a bounded bijective linear map. Then $\Psi$ is right biorthogonality preserving if and only if there is a weak ${ }^{*}$ continuous algebra $*$-isomorphism $\pi: W^{*}(G) \rightarrow W^{*}(H)$ and an invertible element $z$ in $W^{*}(G)$ such that

$$
\Psi^{*}(\pi(x))=z x \quad \text { for all } x \in W^{*}(G) .
$$

\section{Disjointness PRESERVING LineAR MAPs OF Fourier ALGEBRAS}

Let $G$ be a locally compact group with a fixed left Haar measure $m$. Let $C_{00}(G)$ be the subspace of $C_{b}(G)$ consisting of all functions vanishing outside a compact set. Let $L^{p}(G), 1 \leq p<+\infty$, denote the Banach space of $p$-integrable functions with respect to $m$, and let $L^{\infty}(G)$ be the space of essentially bounded measurable functions on $G$ (see, e.g., [29]). Then $G$ is amenable if there exists a positive linear functional $\phi$ of norm one on $C_{b}(G)$ satisfying $\phi(a f)=\phi(f)$ for all $f$ in $C_{b}(G)$ and $a$ in $G$, where $(a f)(t)=f(a t), \forall t \in G$. This is equivalent to the space of bounded left uniformly 
continuous functions $L U C(G)$ has a left translation invariant mean. Here $\operatorname{LUC}(G)$ consists of all those $f$ in $C_{b}(G)$ such that the map $a \mapsto a f$ from $G$ into $C_{b}(G)$ with the norm topology is continuous. See, e.g., [44, 29]. The class of amenable groups includes all solvable groups and all compact groups. On the other hand, the free group on two generators is not amenable, and so is every non-compact connected semisimple Lie group (see, e.g., [46] for details).

For $a$ in $G$, let $\rho(a) \in B\left(L^{2}(G)\right)$, the space of bounded linear operators on $L^{2}(G)$, defined by $\rho(a) h(x)=h\left(a^{-1} x\right)$. Let $V N(G)$ denote the von Neumann algebra in $B\left(L^{2}(G)\right)$ generated by $\{\rho(a): a \in G\}$. The Fourier algebra $A(G)$ of $G$ is the closed linear span of $P(G) \cap C_{00}(G)$ in $B(G)$. Then $A(G)$ can be identified with the unique predual of $V N(G)$ with

$$
\langle f, \rho(a)\rangle=f(a), \quad a \in G, f \in A(G)
$$

also $f \in A(G)$ if and only if there are $\zeta, \eta$ in $L^{2}(G)$ such that

$$
f(x)=\langle\rho(x) \zeta, \eta\rangle_{L^{2}(G)}, \quad \forall x \in G
$$

The Fourier algebra $A(G)$ is a closed ideal in $B(G) \subseteq C_{b}(G)$ with spectrum $G$ given by (11); $A(G)$ has a bounded approximate identity if and only if $G$ is amenable. Furthermore, $A(G)$ has an identity if and only if $G$ is compact. (See [18] and [39] for more details.)

The following elementary result in part (a) is stated in [18, 3.2 and 3.36]. One can adapt a proof for (b) from, for example, [22, Lemma 1], although $G$ is assumed to be abelian there. The part (c) should be known, too. We provide a proof here, as we do not find one handy in the literature.

Lemma 3.1. Let $G$ be a locally compact group.

(a) Let $U$ be an open set in $G$ containing a nonempty compact set $K$. Then there is an $f$ in $A(G)$ such that $f=1$ on $K$ and $f=0$ outside $U$.

(b) Let $\left\{V_{1}, \ldots, V_{n}\right\}$ be an open covering of a compact set $K$ in $G$. Then there exist $f_{1}, \ldots, f_{n}$ in $A(G)$ such that $\sum_{i=1}^{n} f_{i}=1$ on $K$ and $\operatorname{coz}\left(f_{i}\right) \subseteq V_{i}$ for $i=1, \ldots, n$. 
(c) Let $U$ be a neighborhood of a point $x$ in $G$, and $\epsilon>0$. Then there is a $g$ in $A(G)$ and a neighborhood $V$ of $x$ contained in $U$ such that

$$
g=1 \text { on } V, \quad g=0 \text { outside } U, \text { and }\|g\|_{A(G)} \leq 1+\epsilon .
$$

Proof. (c) Without loss of generality, we can assume $x=e$. Let $m$ be the left Haar measure of $G$. Let $W$ be a compact symmetric neighborhood of $e$ such that $e \in W \subseteq$ $W^{3} \subseteq U$. As $0<m(W)<+\infty$, the regularity of $m$ implies that there is an open set $O$ such that $W \subseteq O \subseteq U$ with $m(W) \leq m(O) \leq(1+\epsilon)^{2} m(W)$. By the continuity of the multiplication and the compactness of $W$, there is a neighborhood $V$ of $e$ such that $V \subseteq W$ and $W=e W \subseteq V W \subseteq O$. In particular, $m(W) \leq m(V W) \leq(1+\epsilon)^{2} m(W)$. Define $g=\frac{1}{m(W)} \chi_{V W} * \widetilde{\chi_{W}}$. Then

$$
g(s)=\frac{1}{m(W)} \int_{G} \chi_{V W}(y) \chi_{W}\left(s^{-1} y\right) d m(y)=\frac{m(V W \cap s W)}{m(W)}, \quad \forall s \in G .
$$

We have $g=1$ on $V$ and $g=0$ outside $U$. It follows from [18, Lemma 3.1] that

$$
\|g\|_{A(G)} \leq \frac{1}{m(W)}\left\|\chi_{V W}\right\|_{2}\left\|\chi_{W}\right\|_{2}=\frac{1}{m(W)} \sqrt{m(V W)} \sqrt{m(W)} \leq 1+\epsilon
$$

In what follows, $G$ will denote a locally compact group. Let $x$ be a point in the one-point compactification $G \cup\{\infty\}$ of $G$. Denote by

$$
\begin{aligned}
I_{x} & =\{f \in A(G): f \text { vanishes in a neighborhood of } x\} \\
M_{x} & =\{f \in A(G): f(x)=0\} .
\end{aligned}
$$

Notice that $I_{\infty}=A_{00}(G)$ and $M_{\infty}=A(G)$. Here, $A_{00}(G)$ denotes the algebra of all functions in $A(G)$ with compact supports.

For a function $f$ in $A(G)$, let $\operatorname{coz}(f)$ be the cozero set of $f$, that is,

$$
\operatorname{coz}(f)=\{x \in G: f(x) \neq 0\} .
$$

An open subset $V$ of $G$ is said to be a vanishing set for a linear functional $\varphi$ of $A(G)$ if

$$
\operatorname{coz} f \subseteq V \Longrightarrow \varphi(f)=0, \quad \forall f \in A(G)
$$


We call a linear functional $\varphi$ of $A(G)$ disjointness preserving if

$$
f g=0 \Longrightarrow \varphi(f) \varphi(g)=0, \quad \forall f, g \in A(G) \text {. }
$$

Lemma 3.2. Let $\varphi$ be a nonzero disjointness preserving linear functional of $A(G)$. The following are all equivalent for any $x$ in $G$.

(a) $\varphi_{\mid I_{x}}=0$;

(b) for each open neighborhood $U$ of $x$, there is an $f$ in $A(G)$ with $\varphi(f) \neq 0$ and $\operatorname{coz} f \subseteq U$;

(c) $x \in G \backslash \bigcup\{V \subseteq G: V$ is a vanishing set for $\varphi\}$.

Proof. The equivalence of (b) and (c) is obvious.

For the implication (a) $\Longrightarrow(\mathrm{b})$, suppose $U$ is an open neighborhood of $x$ such that $\varphi(f)=0$ whenever $f$ is in $A(G)$ with coz $f \subseteq U$. Let $V$ be a compact neighborhood of $x$ such that $x \in V \subseteq U$. Let $g$ be in $A(G)$ such that $g=1$ on $V$ and vanishes outside $U$ (Lemma 3.1(a)). Then for all $f$ in $A(G)$, write $f=f g+f(1-g)$. Now coz $f g \subseteq U$ implies $\varphi(f g)=0$. Meanwhile, $f(1-g)_{\mid V}=0$ ensures that $f(1-g) \in I_{x}$ and thus $\varphi(f(1-g))=0$. Hence, we get a contradiction that $\varphi=0$.

Finally, we derive (b) $\Longrightarrow$ (a). Assume $g$ in $A(G)$ vanishes in a neighborhood $U$ of $x$. We may assume that $U$ is open. Hence there is a $f$ in $A(G)$ with $\varphi(f) \neq 0$ and $\operatorname{coz}(f) \subseteq U$. So $f g=0$. As $\varphi$ preserves disjointness, we have $\varphi(g)=0$.

Given a disjointness preserving linear functional $\varphi$ of $A(G)$, we call the set $\operatorname{supp}(\varphi)$ of all points $x$ in $G$ such that one of the equivalent conditions in Lemma 3.2 holds the support of $\varphi$. We also allow $\infty \in \operatorname{supp}(\varphi)$ if $\left.\varphi\right|_{I_{\infty}}=0$.

Lemma 3.3. The support $\operatorname{supp}(\varphi)$ of a nonzero disjointness preserving linear functional $\varphi$ of $A(G)$ consists of exactly one point. That is, there exists a unique $x$ in $G \cup\{\infty\}$ such that $\varphi_{\mid I_{x}}=0$.

Proof. Suppose that $\operatorname{supp}(\varphi) \cap G$ is empty. Then

$$
G=\bigcup\{V \subseteq G: V \text { is a vanishing set for } \varphi\}
$$


Let $f$ be in $A(G)$ with compact support. Then, the compact set $\overline{\operatorname{coz} f}$ can be covered by finitely many vanishing sets of $\varphi$; namely,

$$
\overline{\operatorname{coz}(f)} \subseteq V_{1} \cup \cdots \cup V_{n}
$$

By Lemma 3.1(b), there are $f_{1}, \ldots, f_{n}$ in $A(G)$ such that $\sum_{i=1}^{n} f_{i}=1$ on $\overline{\operatorname{coz}(f)}$ and $\operatorname{coz} f_{i} \subseteq V_{i}$ for $i=1, \ldots, n$. Therefore,

$$
f=\sum_{i=1}^{n} f f_{i} .
$$

Since $\operatorname{coz}\left(f f_{i}\right) \subseteq V_{i}$, we have $\varphi\left(f f_{i}\right)=0$ for $i=1, \ldots, n$. Thus $\varphi(f)=0$ for all $f$ in $A(G)$ with compact support. Therefore, $\left.\varphi\right|_{I_{\infty}}=0$, and thus $\infty \in \operatorname{supp}(\varphi)$.

On the other hand, let us suppose that $r$ and $t$ are distinct members of $\operatorname{supp}(\varphi)$. If $r=\infty$ then the value of $\varphi$ at every function in $A(G)$ supported in a compact neighborhood of $t$ is zero. This conflicts with the assumption that $t$ is a support point of $\varphi$. So we assume none of $r$ and $t$ is the point at infinity. Let $U$ and $V$ be disjoint neighborhoods of $s$ and $t$ in $G$, respectively. Then there are $f$ and $g$ in $A(G)$ such that $\operatorname{coz} f \subseteq U, \operatorname{coz} g \subseteq V$ and both $\varphi(f)$ and $\varphi(g)$ are nonzero. However, this would contradict to the disjointness preserving property of $\varphi$.

Lemma 3.4. If $\varphi$ is a nonzero bounded disjointness linear functional of $A(G)$. Then there is a unique $x$ in $G$ and a nonzero scalar $\lambda$ such that $\varphi=\lambda \delta_{x}$.

Proof. By Lemma 3.3, there is a unique $x$ in $G \cup\{\infty\}$ such that $\varphi_{\mid I_{x}}=0$. Note that the closure $\overline{I_{x}}$ of $I_{x}$ is $M_{x}=\{f \in A(G): f(x)=0\}$ (since $\{x\}$ is a set of spectral synthesis [18]). By the continuity of $\varphi$, its kernel $\operatorname{ker} \varphi \supseteq M_{x}$. This gives $\varphi(f)=0 \Longleftrightarrow f(x)=0$ for all $f$ in $A(G)$. Since $\varphi$ is nonzero, $x \in G$. Consequently, $f=\lambda \delta_{x}$ for some nonzero scalar $\lambda$.

Let $G_{1}, G_{2}$ be locally compact groups. Let $\Psi: A\left(G_{1}\right) \rightarrow A\left(G_{2}\right)$ be a disjointness preserving linear map between two Fourier algebras, that is, $f g=0$ in $A\left(G_{1}\right)$ implies $\Psi(f) \Psi(g)=0$ in $A\left(G_{2}\right)$. For each $s$ in $G_{2}$, let $\Psi^{*} \delta_{s}$ be the disjointness preserving linear functional of $A\left(G_{2}\right)$ defined by

$$
\Psi^{*} \delta_{s}(f)=\Psi(f)(s), \quad \text { for all } f \in A\left(G_{1}\right) .
$$


Denote by

$$
\begin{aligned}
G_{2}^{\circ \circ}=\left\{s \in G_{2}:\right. & \text { there is an } f \text { in } A\left(G_{1}\right) \text { with compact support } \\
& \text { such that } \left.\Psi^{*} \delta_{s}(f)=\Psi(f)(s) \neq 0\right\} \\
=\left\{s \in G_{2}:\right. & \left.\left.\Psi^{*} \delta_{s}\right|_{I_{\infty}} \neq 0\right\} .
\end{aligned}
$$

Lemma 3.5. The set

$\operatorname{supp}\left(\Psi^{*} \delta_{s}\right)=G_{1} \cup\{\infty\} \backslash \bigcup\left\{V \subseteq G_{1} \cup\{\infty\}: V\right.$ is a vanishing set for $\left.\Psi^{*} \delta_{s}\right\}$

is a singleton $\sigma(s)$ for all $s$ in $G_{2}$. Moreover, $s \in G_{2}^{\circ o}$ if and only if $\sigma(s) \in G_{1}$.

Proof. It follows from Lemma 3.3 that $\operatorname{supp}\left(\Psi^{*} \delta_{s}\right)=\{\sigma(s)\}$ is a singleton. Moreover, the first paragraph of the proof of Lemma 3.3 shows that $\sigma(s) \neq \infty$ if $s \in G_{2}^{\circ \circ}$. The converse follows from the definition of $G_{2}^{\circ \circ}$.

Let $\sigma: G_{2} \rightarrow G_{1} \cup\{\infty\}$ be the support map of $\Psi$ as defined in Lemma 3.5. In other words,

$$
\operatorname{supp} \Psi^{*} \delta_{s}=\{\sigma(s)\}, \quad \forall s \in G_{2} .
$$

Lemma 3.6. $\sigma$ is continuous on $G_{2}^{\circ \circ}$.

Proof. Let a net $s_{\alpha} \rightarrow s$ converge in $G_{2}^{\circ \circ}$. Let $G_{1} \cup\{\infty\}$ be the one-point compactification of $G_{1}$. Note that the point $\infty$ at infinity is isolated if and only if $G_{1}$ is compact. Now, $\sigma\left(s_{\alpha}\right)$ has a subnet $\sigma\left(s_{\alpha_{r}}\right) \rightarrow t$ for some $t$ in $G_{1} \cup\{\infty\}$. Suppose that $\sigma(s) \neq t$. Let $U, V$ be disjoint neighborhoods of $t, \sigma(s)$ in $G_{1} \cup\{\infty\}$, respectively. Since $s \in G_{2}^{\circ}$, there is an $f$ in $A\left(G_{1}\right)$ such that $\operatorname{coz} f \subseteq V$ and $\Psi^{*} \delta_{s}(f)=\Psi(f)(s) \neq 0$. By the continuity of $\Psi(f)$ on $G_{2}$ (and hence on $\left.G_{2}^{\circ \circ}\right), \Psi(f)\left(s_{\alpha_{r}}\right) \neq 0$ for large enough $r$. On the other hand, we can also assume $\sigma\left(s_{\alpha_{r}}\right) \in U$. Choose $g$ in $A\left(G_{1}\right)$ such that $\Psi(g)\left(s_{\alpha_{r}}\right) \neq 0$ and cozg $\subseteq U$. It follows from coz $f \cap \operatorname{coz} g=\emptyset$ that $\Psi(f)\left(s_{\alpha_{r}}\right) \Psi(g)\left(s_{\alpha_{r}}\right)=0$, a contradiction.

The following theorem for disjointness preserving linear bijections between Fourier algebras is stated for locally compact amenable groups in a paper of Font [20, Theorem 4] (see also [19]). This is proved by Monfared in [45] without the amenability 
assumptions on the underlying groups, but he assumes the boundedness of the map instead. We drop both the amenability and boundedness assumptions below. Our proof is different from that in [20,45], and inspired by the one given in an earlier paper of Font and Hernández [22] and also by [31, 32].

Theorem 3.7. Let $G_{1}$ and $G_{2}$ be locally compact groups. Let $\Psi: A\left(G_{1}\right) \rightarrow A\left(G_{2}\right)$ be a linear bijection preserving disjointness. Then there exist a non-vanishing continuous bounded scalar function $\lambda$ on $G_{2}$, and a homeomorphism $\sigma$ from $G_{2}$ onto $G_{1}$ such that

$$
\Psi(f)=\lambda \cdot f \circ \sigma, \quad \forall f \in A\left(G_{1}\right) .
$$

In this case, $\Psi$ is automatically bounded in both the uniform and Fourier algebra norms.

Proof. We first recall that the support map $\sigma: G_{2} \rightarrow G_{1} \cup\{\infty\}$ defined as in Lemma 3.5 , by

$$
\operatorname{supp} \Psi^{*} \delta_{x}=\{\sigma(x)\}, \quad \forall x \in G_{2},
$$

which is continuous on $G_{2}^{\circ \circ}$ (Lemma 3.6). It follows from Lemma 3.2 that

$$
\Psi\left(I_{\sigma(x)}\right) \subseteq I_{x}, \quad \forall x \in G_{2} .
$$

Set

$$
\begin{aligned}
& Y_{1}=\left\{y \in G_{2}: \Psi\left(M_{\sigma(y)}\right)=M_{y}\right\}, \\
& Y_{2}=\left\{y \in G_{2}: \Psi\left(M_{\sigma(y)}\right) \neq M_{y}\right\} .
\end{aligned}
$$

Then $G=Y_{1} \cup Y_{2}$ as a disjoint union. Observe that

$$
\begin{aligned}
y \in Y_{1} & \Leftrightarrow \operatorname{ker} \Psi^{*} \delta_{y}=M_{\sigma(y)} \text { is closed } \\
& \Leftrightarrow \Psi^{*} \delta_{y} \text { is }\|\cdot\|_{A\left(G_{1}\right)} \text { bounded } \\
y \in Y_{2} & \Leftrightarrow \Psi^{*} \delta_{y}=\lambda(y) \delta_{\sigma(y)} \text { for some nonzero scalar } \lambda(y) \text { (Lemma 3.4), and } \\
& \quad \Psi_{y} \text { is unbounded. }
\end{aligned}
$$

In this case, the $\|\cdot\|_{\infty}$ and $\|\cdot\|_{A\left(G_{1}\right)}$ boundedness for $\Psi^{*} \delta_{y}$ are equivalent. Moreover,

$$
y \in Y_{1} \Longrightarrow \sigma(y) \neq \infty \quad \Longleftrightarrow \sigma(y) \in G_{1} \Longleftrightarrow y \in G_{2}^{\circ \circ} .
$$

Thus, $G_{2} \backslash G_{2}^{\circ \circ}=\sigma^{-1}(\infty) \subseteq Y_{2}$. 
Define a nonvanishing scalar function $\lambda$ on $Y_{1}$ by $y \mapsto \lambda(y)$ as in (12). In other words, we have

$$
\Psi(f)(y)=\lambda(y) f(\sigma(y)), \quad \forall f \in A\left(G_{1}\right), \forall y \in Y_{1} .
$$

Claim 1. If $\left\{y_{n}\right\}$ is a sequence in $G_{2}$ such that $x_{n}=\sigma\left(y_{n}\right)^{\prime}$ 's are distinct points in $G_{1}$ then

$$
\limsup _{n}\left\|\Psi^{*} \delta_{y_{n}}\right\|<+\infty
$$

Suppose on contrary that $\lim \sup _{n}\left\|\Psi^{*} \delta_{y_{n}}\right\|=+\infty$. Passing to a subsequence, if necessary, we can choose $f_{n}$ in $A\left(G_{1}\right)$ such that

$$
\left\|f_{n}\right\|_{A\left(G_{1}\right)} \leq \frac{1}{n^{3}} \quad \text { but } \Psi\left(f_{n}\right)\left(y_{n}\right)=1, \quad \forall n=1,2, \ldots
$$

We can also assume that the sequence $\left\{x_{n}\right\}$ contains no cluster point of itself. For each $n$, choose a disjoint neighborhood $U_{n}$ of $x_{n}$. By Lemma 3.1(c), we can find a $g_{n}$ in $A\left(G_{1}\right)$ with $\|g\|_{A\left(G_{1}\right)} \leq 2$ such that $g_{n}=1$ on a neighborhood $V_{n}$ of $x_{n}$ contained in $U_{n}$, and $g_{n}=0$ outside $U_{n}$. Since $\left(1-g_{n}\right) f_{n} \in I_{x_{n}}$, we have $\Psi\left(g_{n} f_{n}\right)\left(y_{n}\right)=\Psi\left(f_{n}\right)\left(y_{n}\right)=1$. Since

$$
\sum_{n=1}^{\infty}\left\|n g_{n} f_{n}\right\|_{A\left(G_{1}\right)} \leq \sum_{n=1}^{\infty} \frac{2}{n^{2}}<+\infty
$$

we can define

$$
f=\sum_{n=1}^{\infty} n g_{n} f_{n} \in A\left(G_{1}\right) .
$$

Now $f-n g_{n} f_{n}=0$ on $V_{n}$. Hence, we have $\Psi(f)\left(y_{n}\right)=n \Psi\left(g_{n} f_{n}\right)\left(y_{n}\right)=n$ for $n=1,2, \ldots$ In particular, $\Psi(f)$ is unbounded on $G_{2}$. This contradiction establishes the claim.

Claim 2. The set $\sigma\left(Y_{2}\right)$ consists of at most finitely many points, and the scalar function $\lambda$ is nonvanishing and bounded on $Y_{1}$ by some constant $M>0$.

These assertions follow from Claim 1 and (13).

Claim 3. $Y_{1}$ is closed and $Y_{2}$ is open in $G_{2}$. 
Observe that for any $f$ in $A\left(G_{1}\right)$ we have

$$
\begin{aligned}
\sup \left\{|\Psi(f)|: y \in \overline{Y_{1}}\right\} & =\sup \left\{|\Psi(f)|: y \in Y_{1}\right\} \\
& =\sup \left\{|h(y) \| f(\sigma(y))|: y \in Y_{1}\right\} \\
& \leq M\|f\|_{\infty} \leq M\|f\|_{A\left(G_{1}\right)} .
\end{aligned}
$$

This ensures that $\Psi^{*} \delta_{y}$ is $\|\cdot\|_{A\left(G_{1}\right)}$ bounded for every $y$ in $\overline{Y_{1}}$. By Lemma 3.4, we have $Y_{1}=\overline{Y_{1}}$ is closed. Thus, $Y_{2}=G_{2} \backslash Y_{1}$ is open.

Claim 4. Every point in $\sigma\left(Y_{2}\right)$ is non-isolated in $G_{2} \cup\{\infty\}$.

Let $y \in Y_{2}$. If $\sigma(y)$ is isolated, then $I_{\sigma(y)}=M_{\sigma(y)}$. If $\sigma(y) \neq \infty$ then it follows from

$$
\Psi\left(M_{\sigma(y)}\right)=\Psi\left(I_{\sigma(y)}\right) \subseteq I_{y} \subseteq M_{y}
$$

that $y \in Y_{1}$, a contradiction. If $\sigma(y)=\infty$, we have

$$
A\left(G_{2}\right)=\Psi\left(A\left(G_{1}\right)\right)=\Psi\left(M_{\infty}\right) \subseteq M_{y},
$$

which forces $y=\infty$, a contradiction again as $\infty \notin Y_{2} \subseteq G_{2}$.

Claim 5. $\sigma\left(G_{2}\right)$ is dense in $G_{1}$.

Otherwise, there is a nonzero $f$ in $A\left(G_{1}\right)$ vanishing outside $\overline{\sigma\left(G_{2}\right)}$. In particular,

$$
f \in I_{\sigma(y)}, \forall y \in G_{2} \quad \Longrightarrow \Psi(f) \in I_{y}, \forall y \in G_{2} \quad \Longrightarrow \Psi(f)=0 \quad \Longrightarrow f=0
$$

a contradiction.

It follows from Claims 2, 4 and 5 that

$$
G_{1} \cup\{\infty\}=\overline{\sigma\left(G_{2}\right)} \cup\{\infty\}=\overline{\sigma\left(Y_{1}\right) \cup \sigma\left(Y_{2}\right)} \cup\{\infty\}=\overline{\sigma\left(Y_{1}\right)} \cup\{\infty\} .
$$

It follows from (13) that

$$
\left.\Psi(f)\right|_{Y_{1}}=\left.0 \quad \Longrightarrow \quad f\right|_{\sigma\left(Y_{1}\right)}=0 \quad \Longrightarrow \quad f=0, \quad \forall f \in A\left(G_{1}\right) .
$$

Since $Y_{2}$ is open, $Y_{2}=\emptyset$ by Lemma 3.1(c) and the surjectivity of $\Psi$. Therefore, $G_{2}=Y_{1}=G_{2}^{\circ \circ}$ and $\sigma: G_{2} \rightarrow G_{1}$ is continuous with a dense range.

It then follows from (13) that $\Psi^{-1}$ also preserve disjointness. As a result, there is a continuous map $\tau: G_{1} \rightarrow G_{2}$ and a bounded nonvanishing scalar function $\alpha$ on $G_{1}$ such that

$$
\Psi^{-1}(g)(x)=\alpha(x) g(\tau(x)), \quad \forall x \in G_{1}
$$


Hence,

$$
\begin{aligned}
f(x) & =\Psi^{-1}(\Psi(f))=\alpha(x) \Psi(f)(\tau(x)) \\
& =\alpha(x) \lambda(\tau(x)) f(\sigma(\tau(x))), \quad \forall x \in G_{1}, \quad \forall f \in A\left(G_{1}\right) .
\end{aligned}
$$

This implies

$$
x=\sigma(\tau(x)), \quad \forall x \in G_{1} .
$$

Similarly, we have

$$
y=\tau(\sigma(y)), \quad \forall y \in G_{2} .
$$

It amounts to say that $\sigma$ is a homeomorphism from $G_{2}$ onto $G_{1}$. Furthermore, it is plain that $\lambda$ is continuous on $G_{2}$ by (13) and Lemma 3.1(c).

Finally, it is clear that the uniform norm of $\Psi$ is the bound of $\lambda$. The Fourier algebra norm continuity of $\Psi$ follows from the closed graph theorem. Indeed, if $f_{n} \rightarrow 0$ in $\|\cdot\|_{A\left(G_{1}\right)}$ and $\Psi\left(f_{n}\right) \rightarrow g$ in $\|\cdot\|_{A\left(G_{2}\right)}$ then they also converge in the respective uniform norms. By the uniform continuity of $\Psi$ we have $g=0$.

Remark 3.8. Let $M\left(A\left(G_{2}\right)\right)$ denote the multiplier algebra of $A\left(G_{2}\right)$ consisting of all $u$ in $C_{b}\left(G_{2}\right)$ such that $u g \in A\left(G_{2}\right)$ if $g \in A\left(G_{2}\right)$. Then in Theorem 3.7, $\lambda \in M\left(A\left(G_{2}\right)\right)$. When $G_{2}$ is amenable, $M\left(A\left(G_{2}\right)\right)=B\left(G_{2}\right)$ ([16, Theorem 9]). It follows from (14) that $\lambda$ is invertible in $B\left(G_{2}\right)$. Consequently, the map $f \mapsto f \circ \sigma$ gives rise to an algebra isomorphism from $A\left(G_{1}\right)$ onto $A\left(G_{2}\right)$. Therefore, we can recover the results of Font in [20] by Theorem 3.7.

In a similar manner, we have

Theorem 3.9. Let $G_{1}$ and $G_{2}$ be locally compact groups. Let $\Psi: B\left(G_{1}\right) \rightarrow B\left(G_{2}\right)$ be a linear bijection between the Fourier-Stieltjes algebras preserving disjointness. Then there exist an invertible function $\lambda$ in $B\left(G_{2}\right)$, and a homeomorphism $\sigma$ from $G_{2}$ onto $G_{1}$ such that

$$
\Psi(f)=\lambda \cdot f \circ \sigma, \quad \forall f \in B\left(G_{1}\right) .
$$

In this case, $\Psi$ is automatically bounded in both the uniform and Fourier-Stieltjes algebra norms. Furthermore, $\Psi\left(A\left(G_{1}\right)\right)=A\left(G_{2}\right)$. 
Proof. The arguments in proving Theorem 3.7 can be used in this case too. Note that $\Psi$ will send exactly functions with compact supports to functions with support supports due to (15) and the continuity of $\sigma$ and $\sigma^{-1}$. Since $A\left(G_{1}\right)$ and $A\left(G_{2}\right)$ are closures of functions with compact supports, the last assertion follows by the FourierStieltjes norm continuity of $\Psi$.

\section{Disjointness And orthogonality together DETERMine tOPOLOGICAL} GROUP STRUCTURES

We are now ready to prove our Main Theorem stated in Section 1. More precisely, we provide below a detail description of a bijective linear disjointness preserving map between Fourier algebras, which preserves also some kind of orthogonality. The Main Theorem then becomes a consequence.

Theorem 4.1. Let $G_{1}, G_{2}$ be locally compact groups and $A\left(G_{1}\right), A\left(G_{2}\right)$ the associated Fourier algebras. Let $\Psi: A\left(G_{1}\right) \rightarrow A\left(G_{2}\right)$ be a linear bijection preserving disjointness. Then $\Psi$ is orthogonality decomposition preserving (resp. left, right, left-to-right, or right-to-left biorthogonality preserving) if and only if

(a) there is a positive (resp. nonzero complex) number $\alpha$,

(b) there is a character $\beta$ of the group $G_{2}$,

(c) $w=e_{1}$, the identity element of $G_{1}$ (resp. there is a $w$ in $G_{1}$ ), and

(d) there is a homeomorphic map $\sigma: G_{2} \rightarrow G_{1}$ which is a group isomorphism or antiisomorphism (resp. group isomorphism for left or right biorthogonality preservers, and group anti-isomorphism for left-to-right or right-to-left biorthogonality preservers),

such that for all s in $G_{2}$ we have

$$
\Psi(f)(s)=\alpha \beta(s) f(w \sigma(s))
$$

(resp. $\Psi(f)(s)=\alpha \beta(s) f(\sigma(s) w)$ for left or left-to-right biorthogonality preservers).

The sufficient part is trivial. We shall provide a proof for the necessity part mainly for the case $\Psi$ is an orthogonal decomposition preserver, while the other four cases 
for left, right, left-to-right and right-to-left biorthogonality preservers are similar and easier. We divide the proof into several lemmas.

We first note that by Theorem 3.7, $\Psi$ is automatically bounded with respect to both the uniform norms and the Fourier algebra norms. For each $s$ in $G_{2}$, we denote by $\rho_{s}$ the left translate by $s$, that is,

$$
\rho_{s} h=h\left(s^{-1} t\right), \quad \forall h \in L^{2}(G) .
$$

Note that the set $\left\{\rho_{s}: s \in G_{2}\right\}$ in $\operatorname{VN}\left(G_{2}\right)$ is isomorphic to $G_{2}$ as groups. Moreover,

$$
\left\langle\rho_{s}, u\right\rangle=u(s), \quad \forall u \in A\left(G_{2}\right) .
$$

By Theorem 3.7, we have

$$
\Psi^{*} \rho_{s}=\lambda(s) \rho_{\sigma(s)}, \quad \forall s \in G_{2} .
$$

Here, $\lambda$ is a non-vanishing complex function on $G_{2}$ and $\sigma$ is a homeomorphism from $G_{2}$ onto $G_{1}$. On the other hand, by Proposition 2.1 or Theorem 2.6 (resp. Theorem 2.8 and Remark 2.9 for other one-sided biorthogonality preservers), we also have $\Psi^{*}=z \pi$ for an invertible element $z$ in $\operatorname{VN}\left(G_{1}\right)$ and a Jordan ${ }^{*}$-isomorphism $\pi$ from $\operatorname{VN}\left(G_{2}\right)$ onto $\operatorname{VN}\left(G_{1}\right)$. Let $u$ be the unitary element in $\operatorname{VN}\left(G_{1}\right)$ such that $u z=|z|$. Since $\pi(1)=1$, by $(16)$ we have

$$
z=\Psi^{*}(1)=\Psi^{*}\left(\rho_{e_{2}}\right)=\lambda\left(e_{2}\right) \rho_{\sigma\left(e_{2}\right)},
$$

where $e_{2}$ is the identity element of the group $G_{2}$. It follows that

$$
|z|=u z=\lambda\left(e_{2}\right) u \rho_{\sigma\left(e_{2}\right)} .
$$

Since $|z| \geq 0$, we see that the spectrum of $\lambda\left(e_{2}\right) u \rho_{\sigma\left(e_{2}\right)}$ consists of positive numbers only. However, $u \rho_{\sigma\left(e_{2}\right)}$ is a unitary element in the von Neumann algebra $\operatorname{VN}\left(G_{1}\right)$. This forces the spectrum of $\lambda\left(e_{2}\right) u \rho_{\sigma\left(e_{2}\right)}$ to be a singleton $\{|\alpha|\}$ with $\alpha=\lambda\left(e_{2}\right) \neq 0$. Thus

$$
u \rho_{\sigma\left(e_{2}\right)}=\frac{\bar{\alpha}}{|\alpha|} \rho_{e_{1}},
$$

where $e_{1}$ is the identity element of the group $G_{1}$. Consequently, $u=\frac{\bar{\alpha}}{|\alpha|} \rho_{w}$ where $w=\sigma\left(e_{2}\right)^{-1}$ in $G_{1}$. In the case $\Psi$ is an orthogonal decomposition preserver, $z$ is already positive and thus $u=1, \alpha>0$ and $w=e_{1}$. 
Replacing $\Psi$ by the map $\varphi \rightarrow \Psi(\varphi(u \cdot))$, we can assume $z$ is positive. Note that if the new map carries, by Theorem 3.7 , the form

$$
\Psi(f)(s)=\lambda(s) f(\sigma(s)), \quad \forall f \in A\left(G_{1}\right), \forall s \in G_{2},
$$

then the original map carries the form

$$
f \mapsto \frac{\bar{\alpha}}{|\alpha|} \lambda(s) f(w \sigma(s)), \quad \forall f \in A\left(G_{1}\right), \forall s \in G_{2} .
$$

From now on, we assume the new form and $z \geq 0$. It follows from (17) with $u=1$ that

$$
\alpha>0 \text { and } \sigma\left(e_{2}\right)=e_{1}
$$

Consequently,

$$
z=\alpha \rho_{e_{1}},
$$

and hence,

$$
\Psi^{*}=\alpha \pi,
$$

as $\rho_{e_{1}}$ is the identity of $\operatorname{VN}\left(G_{1}\right)$. By (16), we have

$$
\lambda(s) \rho_{\sigma(s)}=\alpha \pi\left(\rho_{s}\right), \quad \forall s \in G_{2} .
$$

Lemma 4.2. For $s, t$ in $G_{2}$, at least one of the following holds.

(a) $\sigma(s t)=\sigma(s) \sigma(t)$.

(b) $\sigma(s t)=\sigma(t) \sigma(s)$.

Proof. Since $\pi$ is a Jordan $*$-isomorphism, we have

$$
\pi\left(\rho_{s} \rho_{t}+\rho_{t} \rho_{s}\right)=\pi\left(\rho_{s}\right) \pi\left(\rho_{t}\right)+\pi\left(\rho_{t}\right) \pi\left(\rho_{s}\right)
$$

By (18), we have

$$
\alpha\left[\lambda(s t) \rho_{\sigma(s t)}+\lambda(t s) \rho_{\sigma(t s)}\right]=\lambda(s) \lambda(t)\left[\rho_{\sigma(s)} \rho_{\sigma(t)}+\rho_{\sigma(t)} \rho_{\sigma(s)}\right]
$$

or

$$
\alpha \lambda(s t) \rho_{\sigma(s t)}+\alpha \lambda(t s) \rho_{\sigma(t s)}=\lambda(s) \lambda(t)\left[\rho_{\sigma(s) \sigma(t)}+\rho_{\sigma(t) \sigma(s)}\right] .
$$


Suppose on the contrary that $\sigma(s t) \neq \sigma(s) \sigma(t)$ and $\sigma(s t) \neq \sigma(t) \sigma(s)$. If $\sigma(s t)=$ $\sigma(t s)$ then $s t=t s$ since $\sigma$ is a homeomorphism. In this case, by the linear independence of $\rho_{g}$ 's,

$$
\alpha \lambda(s t)=\lambda(s) \lambda(t)=0
$$

This is impossible since $\lambda$ is non-vanishing. Hence $\sigma(s t) \neq \sigma(t s)$. But then $\sigma(s t)$ is distinct from $\sigma(t s), \sigma(s) \sigma(t)$ and $\sigma(t) \sigma(s)$. By (19) and the linear independence, we have again

$$
\alpha \lambda(s t)=0
$$

still a contradiction.

Recall that $\alpha=\lambda\left(e_{2}\right)$, where $e_{2}$ is the identity element of the group $G_{2}$. A character of $G_{2}$ is a unital complex homomorphism of $G_{2}$.

Corollary 4.3. Define $\beta(s)=\lambda(s) / \lambda\left(e_{2}\right)$ for all $s$ in $G_{2}$. Then $\beta$ is a character of the group $G_{2}$, and $\lambda=\alpha \beta$. Moreover,

$$
\pi\left(\rho_{s}\right)=\beta(s) \rho_{\sigma(s)}, \quad \forall s \in G_{2} .
$$

Proof. By (19) and Lemma 4.2, we have

$$
\alpha \lambda(s t)=\lambda(s) \lambda(t), \quad \forall s, t \in G_{2} .
$$

The last assertion follows from (18).

We now follow an idea in [55] (see also [36]). For each $s$ in $G_{2}$, let

$$
\begin{aligned}
& H_{s}=\left\{t \in G_{2}: \sigma(s t)=\sigma(t) \sigma(s)\right\}, \\
& K_{s}=\left\{t \in G_{2}: \sigma(s t)=\sigma(s) \sigma(t)\right\} .
\end{aligned}
$$

Lemma 4.4. Both $H_{s}$ and $K_{s}$ are subgroups of $G_{2}$ for each $s$ in $G_{2}$.

Proof. By a theorem of Kaplansky (see [33, Theorem 5]), the Jordan *-isomorphism $\pi$ can be written as a sum of a $*$-isomorphism $\pi_{1}$ and a $*$-anti-isomorphism $\pi_{2}$. More precisely, there are central projections $p_{i}$ in $\operatorname{VN}\left(G_{i}\right)$ for $i=1,2$ such that

$$
\pi_{1}=\pi_{\mid \mathrm{VN}\left(G_{2}\right) p_{2}}: \mathrm{VN}\left(G_{2}\right) p_{2} \rightarrow \mathrm{VN}\left(G_{1}\right)
$$


is a $*$-isomorphism onto $\operatorname{VN}\left(G_{1}\right) p_{1}$, and

$$
\pi_{2}=\pi_{\mid \mathrm{VN}\left(G_{2}\right)\left(1-p_{2}\right)}: \mathrm{VN}\left(G_{2}\right)\left(1-p_{2}\right) \rightarrow \mathrm{VN}\left(G_{1}\right)
$$

is a $*$-anti-isomorphism onto $\operatorname{VN}\left(G_{1}\right)\left(1-p_{1}\right)$.

Observe that for each $s$ in $G_{2}$

$$
\begin{array}{lll}
t \in H_{s} & \text { if and only if } & {\left[\rho_{s} \rho_{t}-\rho_{t} \rho_{s}\right] p_{2}=0,} \\
t \in K_{s} & \text { if and only if } \quad\left[\rho_{s} \rho_{t}-\rho_{t} \rho_{s}\right]\left(1-p_{2}\right)=0 .
\end{array}
$$

Indeed, suppose $t \in H_{s}$. Then

$$
\sigma(s t)=\sigma(t) \sigma(s)
$$

By (20),

$$
\pi\left(\rho_{s t}\right)=\beta(s t) \rho_{\sigma(s t)}=\beta(s) \beta(t) \rho_{\sigma(t)} \rho_{\sigma_{(s)}} .
$$

In other words,

$$
\pi\left(\rho_{s} \rho_{t}\right)=\pi\left(\rho_{t}\right) \pi\left(\rho_{s}\right)
$$

Hence

$$
\pi\left(\left(\rho_{s} \rho_{t}-\rho_{t} \rho_{s}\right) p_{2}\right)=0
$$

Consequently,

$$
\left(\rho_{s} \rho_{t}-\rho_{t} \rho_{s}\right) p_{2}=0
$$

Conversely, if $t \notin H_{s}$ then $t \in K_{s}$, and thus with a similar argument we have

$$
\pi\left(\rho_{s t}\right)=\pi\left(\rho_{s}\right) \pi\left(\rho_{t}\right) .
$$

It follows

$$
\left(\rho_{s} \rho_{t}-\rho_{t} \rho_{s}\right)\left(1-p_{2}\right)=0
$$

or

$$
\left(\rho_{s} \rho_{t}-\rho_{t} \rho_{s}\right) p_{2}=\rho_{s} \rho_{t}-\rho_{t} \rho_{s}
$$

We are done if the right hand side is not zero. Otherwise, $\rho_{s} \rho_{t}-\rho_{t} \rho_{s}=0$ implies $s t=t s$. Since Jordan $*$-isomorphism preserves commutativity [33, Theorem 5], we then have

$$
\pi\left(\rho_{s}\right) \pi\left(\rho_{t}\right)=\pi\left(\rho_{t}\right) \pi\left(\rho_{s}\right)
$$


By (20), we have

$$
\sigma(s) \sigma(t)=\sigma(t) \sigma(s) .
$$

In particular, $t \in H_{s}$, an absurd.

From (21), it is easy to see that $H_{s}$ is a subgroup of $G_{2}$. The proof that $K_{s}$ is a subgroup of $G_{2}$ is similar.

Lemma 4.5. $\sigma: G_{2} \rightarrow G_{1}$ is either a group isomorphism or anti-isomorphism.

Proof. We note first that for each $s$ in $G_{2}$, either $G_{2}=H_{s}$ or $G_{2}=K_{s}$. By Lemma 4.2, we have $G_{2}=H_{s} \cup K_{s}$. Suppose there were $t_{1}$ in $H_{s} \backslash K_{s}$ and $t_{2}$ in $K_{s} \backslash H_{s}$. It follows that $t_{1} t_{2}$ belongs to either $H_{s}$ or $K_{s}$. Since $t_{1}^{-1} \in H_{s}$ and $t_{2}^{-1} \in K_{s}$, either $t_{2}=t_{1}^{-1}\left(t_{1} t_{2}\right) \in H_{s}$ or $t_{1}=\left(t_{1} t_{2}\right) t_{2}^{-1} \in K_{s}$. This conflict establishes our assertion.

Let now

$$
\begin{aligned}
& H=\left\{s \in G_{2}: H_{s}=G_{2}\right\}, \\
& K=\left\{s \in G_{2}: K_{s}=G_{2}\right\} .
\end{aligned}
$$

It is easy to see that both $H$ and $K$ are subgroups $G_{2}$ and $G_{2}=H \cup K$. Argue similarly as above, we have either $G_{2}=H$ or $G_{2}=K$. This implies that either $\sigma$ is a group anti-isomorphism or isomorphism.

Corollary 4.6. $\pi$ is either a *-isomorphism or a *-anti-isomorphism.

Proof. By Lemma 4.5 and (20), we see that the action of $\pi$ on the linear span of $\left\{\rho_{s}\right.$ : $\left.s \in G_{2}\right\}$ is either multiplicative or anti-multiplicative. Since $\pi$ is weak* continuous and left translations are weak* total in group von Neumann algebras, we thus obtain the result.

Proof of Theorem 4.1. At this point, we have established the proof of Theorem 4.1 for orthogonal decomposition preservers. Note that if $\sigma$ happens to be a group antiisomorphism then $G_{1}$ and $G_{2}$ are isomorphic through the homeomorphism $s \mapsto \sigma(s)^{-1}$.

For left, right, left-to-right and right-to-left biorthogonality preservers, the proofs are easier and go merely the same. We simply mention that in these cases $\pi$ is 
an algebra *-isomorphism for left and right biorthogonality preservers and an algebra *-anti-isomorphism for left-to-right and right-to-left biorthogonality preservers by Theorem 2.8 (see also Remark 2.9), and thus exactly one case of (a) and (b) in Lemma 4.2 always occur. Furthermore, for left and left-to-right biorthogonality preservers, we have $\Psi^{*}(\cdot)=\pi(\cdot) z$ with $z=\Psi^{*}(1)$ instead.

Proof of the Main Theorem. The Fourier algebra case is contained as a part in Theorem 4.1. For the Fourier-Stieltjes algebra case, we shall verify the sufficiency only. By Theorem 3.9, we know that $\Psi$ induces a weighted composition operator $\Phi$ sending $A\left(G_{1}\right)$ onto $A\left(G_{2}\right)$. Since $A\left(G_{i}\right)$ is translation invariant, it is an invariant subspace of $B\left(G_{i}\right)$ as the predual of $W^{*}\left(G_{i}\right)=B\left(G_{i}\right)^{*}$. Hence by [53, Theorem 2.7], there is a central projection $p_{i}$ in $W^{*}\left(G_{i}\right)$ such that $A\left(G_{i}\right)=p_{i} B\left(G_{i}\right)$, where $p_{i} u(x)=$ $u\left(p_{i} x\right), \forall x \in W^{*}\left(G_{i}\right), i=1,2$. Consequently, $\Phi$ also preserves orthogonality (in any of the five senses) as $\Psi$ does. Then the assertion follows from Theorem 4.1.

In Theorem 2.8, we require that both the map $\Psi$ and its inverse $\Psi^{-1}$ are oneside orthogonality preserving. It might be possible that the orthogonality preserving property of $\Psi^{-1}$ can follow from that of $\Psi$. As an evidence, it is the case if the underlying groups are abelian as shown in the following result.

Corollary 4.7. Let $G_{1}$ and $G_{2}$ be two locally compact abelian groups with dual groups $\widehat{G_{1}}$ and $\widehat{G_{2}}$, respectively. Let $\Psi: A\left(G_{1}\right) \rightarrow A\left(G_{2}\right)$ be a bijective linear map, and let $\widehat{\Psi}: L^{1}\left(\widehat{G_{1}}\right) \rightarrow L^{1}\left(\widehat{G_{2}}\right)$ be the associated bijective linear map defined through Fourier transforms. Suppose that both $\Psi$ and $\widehat{\Psi}$ preserves disjointness, i.e.,

$$
\begin{array}{ccc}
f g=0 \text { in } A\left(G_{1}\right) & \Longrightarrow & \Psi(f) \Psi(g)=0 \text { in } A\left(G_{2}\right), \text { and } \\
\widehat{f} \widehat{g}=0 \text { in } L^{1}\left(\widehat{G_{1}}\right) \quad \Longrightarrow & \widehat{\Psi}(\widehat{f}) \widehat{\Psi}(\widehat{g})=0 \text { in } L^{1}\left(\widehat{G_{2}}\right) .
\end{array}
$$

Then $G_{1}$ and $G_{2}$ are isomorphic as topological groups. More precisely, there is a nonzero complex number $\alpha$, a character $\beta$ of $G_{2}$, an element $w$ in $G_{2}$, and a topological group isomorphism $\sigma: G_{2} \rightarrow G_{1}$ such that

$$
\Psi(f)(s)=\alpha \beta(s) f(w \sigma(s)), \quad \forall f \in A\left(G_{1}\right), s \in G_{2} .
$$


Proof. By Theorem 3.7, $\Psi$ is automatically bounded.

Assume first that the dual group $\widehat{G_{i}}$ of $G_{i}$ is $\sigma$-compact for $i=1,2$. Recall that $A\left(G_{i}\right)$ is isomorphic to the algebra $L^{1}\left(\widehat{G_{i}}\right)$ of integrable functions with respect to a fixed $\sigma$-finite (left) Haar measure, equipped with the convolution product, via the inverse Fourier transform $f \mapsto \widehat{f}$. In this setting, $f, g$ in $A\left(G_{i}\right)$ are orthogonal as normal functionals of $V N\left(G_{i}\right) \cong L^{\infty}\left(\widehat{G_{i}}\right)$ if and only if $\widehat{f} \widehat{g}=0$ in $L^{1}\left(\widehat{G_{i}}\right)$, i.e., $\widehat{f}, \widehat{g}$ have disjoint cozero sets in $\widehat{G_{i}}$. The condition (23) amounts to say that $\Psi$ is orthogonality preserving.

On the other hand, noting that $\widehat{G_{1}}$ is $\sigma$-compact, we can apply [40, Corollary 5.6] to the bounded disjointness preserving linear map $\widehat{\Psi}$ to get a measurable function $h$ on $\widehat{G_{2}}$ and a $\mathcal{B}_{2}^{*}$-measurable transformation $\phi: \widehat{G_{2}} \rightarrow \widehat{G_{1}}$ such that

$$
\widehat{\Psi}(\widehat{f})=h \widehat{f} \circ \phi \quad \text { almost everywhere on } \widehat{G_{2}}, \quad \forall \widehat{f} \in L^{1}\left(\widehat{G_{1}}\right) .
$$

Here, $\mathcal{B}_{2}^{*}$ is the (measure) completion of the Borel $\sigma$-algebra $\mathcal{B}_{2}$ of $\widehat{G_{2}}$ with respect to the Haar measure. By the injectivity of $\widehat{\Psi}$, we see that $\widehat{G_{1}} \backslash \phi\left(\widehat{G_{2}}\right)$ is of measure zero. Moreover, if $z(h)$ is the zero set of $h$ in $\widehat{G_{2}}$ then the set $\phi^{-1}(z(h))$ in $\widehat{G_{1}}$ is of measure zero. Suppose $\widehat{\Psi}(\widehat{f}) \widehat{\Psi}(\widehat{g})=0$ for some $\widehat{f}, \widehat{g}$ in $L^{1}\left(\widehat{G_{1}}\right)$. It follows from (25) that $h^{2}(\widehat{f} \circ \phi)(\widehat{g} \circ \phi)=0$, and thus $\widehat{f} \widehat{g}=0$, almost everywhere on $\widehat{G_{1}}$. Hence, $\widehat{\Psi}^{-1}$ preserves disjointness, or equivalently, $\Psi^{-1}$ also preserves orthogonality.

In general, if $\widehat{G_{1}}$ is not $\sigma$-compact, let $K$ be a compact symmetric neighborhood of the identity and let $H=\bigcup_{n=1}^{\infty} K^{n}$. Then $H$ is a $\sigma$-compact clopen subgroup of $\widehat{G_{1}}$. We can write $\widehat{G_{1}}$ as a (possibly uncountable) disjoint union of cosets $g_{\lambda} H$ of $H$, each of which is $\sigma$-compact and clopen. For every function $\widehat{f}$ in $L^{1}\left(\widehat{G_{1}}\right)$, we have

$$
\|\widehat{f}\|_{L^{1}\left(\widehat{G_{1}}\right)}=\sum_{n}\left\|\left.\widehat{f}\right|_{g_{\lambda_{n}} H}\right\|_{L^{1}\left(g_{\lambda_{n}} H\right)}
$$

for an at most countably subfamily of these cosets. Consequently,

$$
L^{1}\left(\widehat{G_{1}}\right) \cong \ell^{1}-\bigoplus_{\lambda} L^{1}\left(g_{\lambda} H\right)
$$

Therefore,

$$
V N\left(G_{1}\right) \cong L^{1}\left(\widehat{G_{1}}\right)^{*} \cong \ell^{\infty}-\bigoplus_{\lambda} L^{\infty}\left(g_{\lambda} H\right)
$$


Note that due to the left translation invariance of Haar measures, every $L^{1}\left(g_{\lambda} H\right) \cong$ $L^{1}(H)$ and $L^{\infty}\left(g_{\lambda} H\right) \cong L^{\infty}(H)$. In this setting, we see again that $f, g$ in $A\left(G_{1}\right)$ are orthogonal as normal functionals of $V N\left(G_{1}\right)$ if and only if $\widehat{f} \widehat{g}=0$ in $L^{1}\left(\widehat{G_{1}}\right)$. We can decompose $L^{1}\left(\widehat{G_{2}}\right)$ in a similar way. So $(23)$ says that $\Psi$ preserves orthogonality.

We check that $\widehat{\Psi}^{-1}$ also preserves disjointness. Suppose $f, g$ be in $A\left(G_{1}\right)$ such that $\widehat{\Psi}(\widehat{f}) \widehat{\Psi}(\widehat{g})=0$. Observing $(26)$, we can assume that there is a $\sigma$-compact Borel subset $X$ of $\widehat{G_{1}}$ such that $\widehat{f}, \widehat{g} \in L^{1}(X)$. Here, $X$ is a disjoint union of at most countably many cosets of $H$. Since the bounded bijective linear map $\widehat{\Psi}$ preserves disjointness, the induced map $\widehat{\Psi}_{X}: L^{1}(X) \rightarrow L^{1}\left(\widehat{G_{2}}\right)$ is also bounded, injective, and preserves disjointness. It follows from an argument similar to the case $G_{1}$ being $\sigma$-compact above that

$$
\widehat{\Psi}_{X}(\widehat{f}) \widehat{\Psi}_{X}(\widehat{g})=\widehat{\Psi}(\widehat{f}) \widehat{\Psi}(\widehat{g})=0 \quad \Longrightarrow \quad \widehat{f} \widehat{g}=0
$$

Hence, $\widehat{\Psi}^{-1}$ also preserves disjointness, and thus $\Psi$ is biorthogonality preserving. Note that all one-side orthogonality preservers coincide in this case. The assertions follow from Theorem 4.1.

When $G$ is a compact abelian group, we can perform convolutions in the Fourier algebra $A(G)$, as it is contained in $L^{1}(G)$. Applying Fourier and inverse Fourier transforms we see that the condition (23) in Corollary 4.7 and the condition (27) below are equivalent.

Corollary 4.8. Let $G_{1}, G_{2}$ be compact abelian groups. Suppose a bijective linear map $\Psi: A\left(G_{1}\right) \rightarrow A\left(G_{2}\right)$ preserves both zero pointwise products and zero convolution products, i.e.,

$$
\begin{gathered}
f g=0 \text { in } A\left(G_{1}\right) \quad \Longrightarrow \quad \Psi(f) \Psi(g)=0 \text { in } A\left(G_{2}\right), \text { and } \\
f * g=0 \text { in } A\left(G_{1}\right) \quad \Longrightarrow \quad \Psi(f) * \Psi(g)=0 \text { in } A\left(G_{2}\right) .
\end{gathered}
$$

Then $G_{1}$ and $G_{2}$ are isomorphic as topological groups, and the conclusions in Corollary 4.7 hold.

To end this section, we would like to mention a recent work of Lin [43]. Assume $G_{1}$ and $G_{2}$ are locally compact amenable groups. Using operator space structure of the 
Fourier algebras [50, 17, 24, 30], Lin shows that every completely bounded surjective disjointness preserving linear map $\Psi: A\left(G_{1}\right) \rightarrow A\left(G_{2}\right)$ carries the form $\Psi(f)(y)=$ $\lambda(y) f(\sigma(y))$. Here, $\lambda$ is invertible in $B\left(G_{2}\right)$, and $\sigma: G_{2} \rightarrow G_{1}$ is a continuous piecewise affine proper map. Therefore, $\sigma$ induces a topological group isomorphism when $\Psi$ is bijective. Since algebra *-isomorphisms and multiplications are completely bounded, together with Theorem 2.8 Lin's results apply in some cases of Theorem 4.1 concerning one-side biorthogonality preservers, provided that the underlying groups are amenable. However, as pointed out in [30, Proposition 3.8], there are completely bounded homomorphisms between non-amenable locally compact groups, which do not give rise to piecewise affine maps between the underlying groups. Thus, Lin's results do not help when the amenability assumption is absent.

\section{UNBOUNDED DISJOINTNESS PRESERVING LINEAR FUNCTIONALS}

This section is devoted to the study of unbounded disjointness preserving linear functionals on the Fourier algebra $A(G)$ of a locally compact group $G$. As we shall see, there are many of them.

Lemma 5.1 ([10, Lemma 2.7]; see also [31] or [2, Lemma 2.7]). Let $\mathfrak{F}$ be any free ultrafilter on $\mathbb{N}$. Then the algebraic dimension of the quotient space

$$
\ell_{\infty} /\left\{y \in \ell_{\infty}: y \text { vanishes on an element of } \mathfrak{F}\right\}
$$

is at least the continuum.

Lemma 5.2. Let $G$ be a locally compact group. If $I_{x}=M_{x}$ for any $x$ in $G$, then $G$ is a discrete group. Indeed, when $G$ is not discrete, the dimension of the vector space $M_{x} / I_{x}$ is at least the continuum.

Proof. Let $K$ be a compact neighborhood of the identity element $e$ in $G$. If $G$ is not discrete then $K$ contains an infinite sequence $\left\{x_{n}\right\}_{n}$ of distinct points. By translating, we can assume $x$ is a cluster point of $\left\{x_{n}\right\}_{n}$.

We make an elementary observation. 
Claim. $x$ is the unique point in $G$ satisfying that every neighborhood of $x$ contains all but finitely many $x_{n}$ 's.

Indeed if $z$ is another such point, then each neighborhood of $z$ contains all but finitely many $x_{n}$ 's and thus intersects with every neighborhood of $x$. But $G$ is Hausdorff, and hence $x=z$.

It follows from the Claim that, by passing to a subsequence if necessary, we can choose an infinite disjoint sequence $\left\{V_{n}\right\}$ of compact neighborhoods of $x_{n}$ for $n \geq 1$.

For each $n \geq 1$, let $f_{n} \in A(G)$ such that $0 \leq f_{n} \leq 1, f_{n}\left(x_{n}\right)=1, \operatorname{coz} f_{n} \subseteq V_{n}$ and $\left\|f_{n}\right\|_{A(G)} \leq 2$ (Lemma 3.1(c)). Let $U_{n}=\operatorname{coz}_{n} \subseteq V_{n}$ for $n=1,2, \ldots$. Construct an ultrafilter $\mathfrak{F}$ on $\mathbb{N}$ as follows.

$A \in \mathfrak{F} \quad$ if and only if there is a neighborhood $U$ of $x$ intersecting exactly those $U_{n}$ with $n$ in $A$.

Then $\mathfrak{F}$ is free since it contains no finite subsets of $\mathbb{N}$; for else $x$ would not be a cluster point of $\left\{x_{n}\right\}_{n}$. For any bounded sequence $y=\left(y_{n}\right)$ in $\ell_{\infty}$, define $f_{y}=$ $\sum_{n=1}^{\infty} y_{n} f_{n} / 2^{n} \in A(G)$. Then $f_{y}(x)=0$. Note that $f_{y}$ vanishes in any neighborhood of $x$, if and only if, the set $A=\left\{n \in \mathbb{N}: y_{n}=0\right\}$ belongs to $\mathfrak{F}$. Consequently,

$$
\operatorname{dim} M_{x} / I_{x} \geq \operatorname{dim}\left(\ell_{\infty} /\left\{y \in \ell_{\infty}: y \text { vanishes on an element of } \mathfrak{F}\right\}\right)
$$

which is at least the continuum by Lemma 5.1 .

Let $S$ be an ideal of a commutative ring $R$. The radical of $S$ is defined to be

$$
r(S)=\left\{r \in R: r^{n} \in S \text { for some } n=1,2, \ldots\right\} .
$$

The following elementary lemma can be found in a standard algebra textbook, e.g., [8].

Lemma 5.3. $S=r(S)$ if and only if $S$ is the intersection of all prime ideals of $R$ containing it.

Lemma 5.4. Let $G$ be a locally compact non-discrete group $G$ and $x$ be in $G$. Then there is a prime ideal $P$ of $A(G)$ such that

$$
I_{x} \subset P \subset M_{x}
$$


where all inclusions are strict.

Proof. Note first that $M_{x}$ is the unique maximal ideal of $A(G)$ containing $I_{x}$. Since $r\left(I_{x}\right)=I_{x}$, we have by Lemma 5.3 that

$$
I_{x}=\bigcap\left\{P: P \text { is a prime ideal of } A(G) \text { containing } I_{x}\right\} .
$$

By Lemma 5.2, $I_{x} \neq M_{x}$. It follows that there is some prime ideal $P$ of $A(G)$ lying strictly in between $I_{x}$ and $M_{x}$.

Lemma 5.5. Let $G$ be a locally compact group. Let $\varphi$ be a linear functional of $A(G)$. Then $\varphi$ is disjointness preserving if $\operatorname{ker} \varphi$ contains a prime ideal $P$ of $A(G)$.

Proof. Let $f g=0$ in $A(G)$. Since $P$ is prime, at least one of $f, g$ belongs to $P$. Thus $\varphi(f)=0$ or $\varphi(g)=0$.

Theorem 5.6. Let $G$ be a locally compact infinite group and $f$ in $A(G)$ with infinite support. Then there is an unbounded disjointness preserving linear functional $\varphi$ of $A(G)$ such that $\varphi(f) \neq 0$. Indeed, we have at least $\mathfrak{c}$ linearly independent choices of such $\varphi$, where $\mathfrak{c}$ is the cardinality of the continuum.

Proof. Let $x_{n}$ in $G$ be distinct such that $f\left(x_{n}\right) \neq 0$ for $n=1,2, \ldots$

Suppose first that $G$ is not discrete and $\left\{x_{n}\right\}_{n}$ has a cluster point in $G$. By Lemma 5.4, there is a prime ideal $P$ of $A(G)$ such that the following strict inclusions hold

$$
I_{x} \subset P \subset M_{x} .
$$

We can also assume $f \notin P$. Let $\varphi$ be any linear functional vanishing on $P$ but not on $M_{x}$ and $f$. Since $\{x\}$ is a set of spectral synthesis, we have $\overline{I_{x}}=M_{x}$. Consequently, $\varphi$ is unbounded as its kernel is not closed. By Lemma 5.5, $\varphi$ is disjointness preserving.

To enumerate all such $\varphi$, let $\left\{P_{\alpha}\right\}_{\alpha}$ be a chain of prime ideals containing $I_{x}$ but not $f$ and ordered by theoretical set inclusion. Let $\aleph$ be the cardinality of this chain. When $\aleph$ is less than the continuum, one of the quotient space $M_{x} / P_{\alpha}$ must have dimension at least the continuum by Lemma 5.2. Conversely, $\aleph$ must be at least the continuum when all $M_{x} / P_{\alpha}$ have dimension less than the continuum. In both situations, we will have at least $\mathfrak{c}$ linear independent choices of $\operatorname{such} \varphi$. 
For the case $x_{n} \rightarrow \infty$ (this always happens when $G$ is discrete), using the argument in the proof of Lemma 5.2 we see that the dimension of $A(G) / A_{00}(G)$ is at least the continuum. Then a similar reasoning as above will give us again at least the continuum many linearly independent choices of unbounded disjointness preserving linear functionals $\varphi$ of $A(G)$ with $\varphi(f) \neq 0$.

\section{Disjointness preserving Linear functionals on $L U C(S)$}

A semitopological semigroup is a semigroup $(S, \cdot)$, or simply $S$, with a Hausdorff topology such that for each $a$ in $S$ the mappings $s \mapsto s \cdot a$ and $s \mapsto a \cdot s$ from $S$ into $S$ are continuous. Let $C_{b}(S)$ denote the $\mathrm{C}^{*}$-algebra of bounded complexvalued functions $f: S \rightarrow \mathbb{C}$ with norm $\|f\|_{\infty}=\sup \{|f(x)|: x \in S\}$. Let $\operatorname{LUC}(S)$ denote the $\mathrm{C}^{*}$-algebra of all $f$ in $C_{b}(S)$ such that the mapping $s \mapsto \ell_{s} f$ from $S$ into $\left(C_{b}(S),\|\cdot\|_{\infty}\right)$ is continuous, where $\left(\ell_{s} f\right)(t)=f(s t), t \in S$. When $S$ is a topological group, $L U C(S)$ is precisely the space of bounded left uniformly continuous functions on $S$ (see, e.g., $[44,29])$. Then $L U C(S)$ is a $\mathrm{C}^{*}$-subalgebra of $C_{b}(S)$ invariant under left and right translations containing constants. $S$ is called extremely left amenable if there is a multiplicative linear functional $m$ in $\operatorname{LUC}(S)^{*}, m \geq 0,\|m\|=1$, and $\left\langle m, \ell_{s} f\right\rangle=\langle m, f\rangle$ for all $s$ in $S$ and $f$ in $L U C(S)$. When $S$ is discrete, $S$ is extremely left amenable if and only if whenever $a, b \in S$ there exists $c$ in $S$ such that $a c=b c=c$, i.e., any two elements in $S$ has a common right zero divisor (see [26], and also [38] for a different proof). In particular, if $S$ is right cancellative, then $S$ must be trivial. Also if $G$ is a locally compact group, then $G$ is extremely left amenable exactly when $G$ is trivial (see [27]). On the other hand, there are important amenable topological groups $G$ such as $\mathcal{U}\left(\ell_{2}\right)$, the group of unitary operators on the Hilbert space $\ell_{2}$ with the strong operator topology, or more generally, Levy groups (see [28, 37], or the beautiful monograph of V. Pestov [48] for more details).

As shown by T. Mitchell [44], a semitopological semigroup $S$ is extremely left amenable if and only if $S$ has the following fixed point property: whenever $S$ acts on a compact Hausdorff space $X$ such that the mapping $S \times X \rightarrow X,(s, x) \mapsto s x$, $s \in S, x \in X$, is jointly continuous, then $X$ contains a common fixed point for $S$. 
The following is a new characterization of extremely left amenable semitopological semigroups in terms of disjoint preserving functionals.

Proposition 6.1. Let $S$ be a semitopological semigroup. Then $S$ is extremely left amenable if and only if there is a nonzero disjointness preserving linear functional $\varphi$ on $\operatorname{LUC}(S)$ such that $\operatorname{ker} \varphi$ is left translation invariant.

Proof. One direction is clear. Assume $\operatorname{ker} \varphi$ is left translation invariant, let $x \in X$, the spectrum of $\operatorname{LUC}(S)$ such that $\left.\varphi\right|_{I_{x}}=0$ (as in Lemma 3.3). Then $I_{s x} \subseteq \operatorname{ker} \varphi$, where $s x \in X$ defined by $\langle s x, f\rangle=\left\langle x, \ell_{s} f\right\rangle$ for all $s$ in $S$ and $f$ in $L U C(S)$ by the left translation invariance of $\varphi$. Since $x$ is unique, $s x=x$ for all $s$ in $S$. In particular, $S$ is extremely left amenable.

Corollary 6.2. Let $G$ be a locally compact group. If $L U C(G)$ has a nonzero disjointness preserving linear functional $\varphi$ such that $\operatorname{ker} \varphi$ is left translation invariant, then $G$ is trivial.

Proof. This follows from Proposition 6.1 and [27].

Let $G$ be a topological group, and $A P(G)$ be the $\mathrm{C}^{*}$-subalgebra of $C_{b}(S)$ consisting of all almost periodic functions on $G$, i.e., those $f$ in $C_{b}(G)$ such that $\mathcal{L} O(f)=\left\{\ell_{a} f\right.$ : $a \in G\}$ is relatively compact in the norm topology of $C_{b}(G)$. As a well known fact, $f \in A P(G)$ if and only if $\mathcal{R} O(f)=\left\{r_{a} f: a \in G\right\}$ is relatively compact in the norm topology of $C_{b}(G)$, where $\left(r_{a} f\right)(t)=f(a t), t \in G$.

Proposition 6.3. Let $G$ be a topological group. If $A P(G)$ has a disjointness preserving linear functional $\varphi: A P(G) \rightarrow \mathbb{C}$ such that $\operatorname{ker} \varphi$ is left translation invariant then $A P(G)=\mathbb{C}$.

Proof. Let $\Lambda$ denote the spectrum of the $\mathrm{C}^{*}$-algebra $A P(G)$, and $m_{0} \in \Lambda$ such that $\operatorname{ker} \varphi \subseteq \operatorname{ker} m_{0}$. Then $\Lambda$ is a compact topological group with multiplication defined by

$$
\langle m \cdot n, f\rangle=\langle m, n \cdot f\rangle, \quad \forall m, n \in \Lambda, f \in A P(G)
$$


where

$$
n \cdot f(g)=\left\langle n, \ell_{g} f\right\rangle, \quad \forall g \in G \text {. }
$$

Consequently,

$$
\hat{g} \cdot m_{0}=m_{0}, \quad \forall g \in G
$$

where $\hat{g}$ in $\Lambda$ is defined by

$$
\langle\hat{g}, f\rangle=f(g), \quad \forall f \in A P(G) .
$$

But $\{\hat{g}: g \in G\}$ is dense in $\Lambda$. Hence $m \cdot m_{0}=m_{0}$ for all $m$ in $\Lambda$. Since $\Lambda$ is a group, $\Lambda$ must be trivial.

\section{REFERENCES}

[1] Y. A. Abramovich, Multiplicative representations of disjointness preserving operators, Indag. Math. 45 (1983), 265-279.

[2] Y. A. Abramovich and Z. Lipecki, On ideals and sublattices in linear lattices and F-lattices, Math. Proc. Camb. Phil. Soc. 108 (1990), 79-87.

[3] C. D. Aliprantis and O. Burkinshaw, Positive operators, Academic Press, Orlando, 1985.

[4] H. Araki, An application of Dye's Theorem on projection lattices in orthogonally decomposable isomorphisms, Pac. J. Math. 37 (1989), 1-13.

[5] W. Arendt, Spectral properties of Lamperti operators, Indiana Univ. Math. J., 32 (1983), 199-215.

[6] W. Arendt and J. de Cannière, Order isomorphisms of Fourier algebras, J. Funct. Anal. 50 (1983), 1-7.

[7] W. Arendt and J. de Cannière, Order isomorphisms of Fourier-Stieltjes algebras, Math. Ann. 263 (1983), 145-156.

[8] N. F. Atiyah and I. G. Macdonald, Introduction to commutative algebra, Addison-Wesley Publishing Co., Reading, 1969.

[9] L. G. Brown, Semicontinuity and multipliers of $C^{*}$-algebras, Can. J. Math. XL(4) (1988), 865-988.

[10] L. G. Brown and N.-C. Wong, Unbounded disjointness preserving linear functionals, Monatshefte für Mathematik 141(1) (2004), 21-32.

[11] L. J. Bunce and J. D. M. Wright, On orthomorphisms between von Neumann preduals and a problem of Araki, Pac. J. Math. 158(2) (1993), 265-272.

[12] M. Burgos, F. J. Fernandez-Polo, J. J. Garces, J. M. Moreno and A. M. Peralta, Orthogonality preservers in $\mathrm{C}^{*}$-algebras, $\mathrm{JB}^{*}$-algebras and $\mathrm{JB}^{*}$-triples, J. Math. Anal. Appl., 348 (2008), 220-233.

[13] M. Burgos, F. J. Fernandez-Polo, J. J. Garces and A. M. Peralta, Orthogonality preservers revisited, AsianEur. J. Math. 2(3) (2009), 387-405.

[14] M. Burgos, J. J. Garces and A. M. Peralta, Automatic continuity of biorthogonality preservers between compact $C^{*}$-algebras and von Neumann algebras, J. Math. Anal. Appl. 376(1) (2011), 221-230.

[15] M. A. Chebotar, W.-F. Ke, P.-H. Lee and N.-C. Wong, Mappings preserving zero products, Studia Math. 155(1) (2003), 77-94.

[16] A. Derighetti, Some results on the Fourier-Stieltjes Algebra of a locally compact group, Comment. Math. Helv. 45 (1970), 219-228. 
[17] E. G. Effros and Z. J. Ruan, Operator Spaces, London Math. Soc. Monogr. Ser. vol. 23, Oxford Science Publications, New York, 2000.

[18] P. Eymard, L'Algèbre de Fourier d'un groupe localement compact, Bull. Soc. Math. France 92 (1964), 181-236.

[19] J. J. Font, Automatic continuity of certain isomorphisms between regular Banach function algebras, Glasgow Math. J. 30 (1997), 333-343.

[20] J. J. Font, Disjointness preserving mappings between Fourier algebras, Colloquium Math. 77(2) (1998), 179-187.

[21] J. J. Font and S. Hernandez, On separating maps between locally compact spaces, Arch. Math. (Bessel) 63 (1994), 158-165.

[22] J. J. Font and S. Hernandez, Automatic continuity and representation of certain linear isomorphisms between group algebras, Indag. Mathem. N. S. 6(4) (1995), 397-409.

[23] J. J. Font and S. Hernandez, Algebraic characterizations of locally compact groups, J. Austral. Math. Soc. (Series A) 62 (1997), 405-420.

[24] B. E. Forrest and V. Runde, Amenability and weak amenability of the Fourier algebra, Math. Z. 250 (2005), 731-744.

[25] H.-L. Gau, J.-S. Jeang and N.-C. Wong, A Banach-Stone theorem for separating linear bijections, Taiwanese J. Math. 6(3) (2002), 399-403.

[26] E. Granirer, Extremely amenable semigroups I, Math. Scand. 17 (1965), 177-197.

[27] E. Granirer and A. T.-M. Lau, Invariant means on locally compact groups, Illinois J. Math. 15 (1971), 249-257.

[28] M. Gromov and V. D. Milman, A topological application of the isoperimetric inequality, Amer. J. Math. 105 (1983), 843-854.

[29] E. Hewitt and K. Ross, Abstract harmonic analysis I, 2nd edition, Springer-Verlag, 1979.

[30] M. Ilie and N. Spronk, Completely bounded homomorphisms of the Fourier algebras, J. Funct. Anal. 225(2) (2005), 480-499.

[31] K. Jarosz, Automatic continuity of separating linear isomorphisms, Can. Math. Bull. 33 (1990), $139-144$.

[32] J.-S. Jeang and N.-C. Wong, Weighted composition operators of $C_{0}(X)$ 's, J. Math. Anal. Appl. 201 (1996), 981-993.

[33] R. V. Kadison, Isometries of operator algebras, Ann. of Math. 54 (1951), 325-338.

[34] I. Kaplansky, Lattices of continuous functions, Bull. Amer. Math. Soc. 53 (1947), 617-623.

[35] J. Lamperti, On the isometries of certain function-spaces, Pacific J. Math. 8 (1958), 459-466.

[36] A. T.-M. Lau, The Fourier-Stieltjes algebra of a topological semigroup with involution, Pacific J. Math. 77 (1978), no. 1, 165-181.

[37] A. T.-M. Lau and J. Ludwig, Fourier-Stieltjes algebra of a topological group, Adv. Math. 229 (2012), no. 3, 2000-2023.

[38] A. T.-M. Lau and Yong Zhang, Fixed point properties for semigroups of nonlinear mappings and amenability, J. Funct. Anal. 263 (2012), 2949-2977.

[39] H. Leptin, Sur l'alge'bre de Fourier d'un groupe localement compact, C. R. Acad. Sci. Paris Se'r. A-B 266 (1968), A1180-A1182.

[40] F. Lessard, Linear operators induced by point transformation, Ann. Sci. Math. Québec 18(1) (1994), 79-93.

[41] C.-W. Leung, C.-W. Tsai and N.-C. Wong, Linear disjointness preservers of W*-algebras, Math. Z. 270 (2012), no. 3-4, 699-708.

[42] B. Li, Introduction to operator algebras, World Scienctific Publ. Co. Singapore, 1992.

[43] Y.-F. Lin, Completely bounded disjointness preserving operators between Fourier algebras, J. Math. Anal. Appl. 382(1) (2011), 469-473.

[44] T. Mitchell, Topological semigroups and multiplicative left invariant means, Trans. Amer. Math. Soc. 122 (1966), 195-202. 
[45] M. S. Monfared, Extensions and isomorphisms for the generalized Fourier algebras of a locally compact group. J. Funct. Anal. 198(2) (2003), 413-444.

[46] A. L. T. Paterson, Amenability, Mathematical Surveys and Monographs, 29, American Math. Soc. Providence, RI, 1988.

[47] G. K. Pedersen, C*-algebras and their automorphism groups, Academic Press, New York, 1979.

[48] V. Pestov, Dynamics of infinite-dimensional Groups. A Ramsey-Dvoretzky-Milman Phenomenon, University Lecture Series 40, Amer. Math. Soc. 2006.

[49] H. L. Royden, Real Analysis, 3rd ed. New York, Macmillan, 1988.

[50] Z.-J. Ruan, The operator amenability of $A(G)$, Amer. J. Math. 117(6) (1995), 1449-1474.

[51] J. Schweizer, Interplay between noncommutative topology and operators on $\mathrm{C}^{*}$-Algebras, $\mathrm{PhD}$ Dissertation, Eberhard-Karls-Universitat, Tubingen, Germany (1997).

[52] E. Størmer, On the Jordan structure of $\mathrm{C}^{*}$-algebras, Trans. Amer. Math. Soc. 120(3), 1965, $438-447$.

[53] M. Takesaki, Theory of operator algebras I, Springer-Verlag, New York-Heidelberg, 1979.

[54] J. Vesterstrøm, On the homomorphic image of the center of a $\mathrm{C}^{*}$-algebra, Math. Scand. 29 (1971), 134-136.

[55] M. E. Walter, W*-algebras and Nonabelian Harmonic Analysis, J. Funct. Anal. 11 (1972), $17-38$.

[56] M. Wolff, Disjointness preserving operators on $C^{*}$-algebras, Arch. Math. 62 (1994), 248-253.

[57] N.-C. Wong, Triple homomorphisms of operators algebras, Southeast Asian Bulletin of Math. 29 (2005), 401-407.

[58] N.-C. Wong, Zero product preservers of C*-algebras, Contemporary Math. 435 (2007), 377-380.

Department of Mathematical and Statistical Sciences, University of Alberta, Edmonton, Alberta, Canada T6G-2G1

E-mail address: tlau@math.alberta.ca

Department of Applied Mathematics, National Sun Yat-sen University, Kaohsiung, 80424, TAIWAN.

E-mail address: wong@math.nsysu.edu.tw 